\title{
Space Mapping With Adaptive Response Correction for Microwave Design Optimization
}

\author{
Koziel, S.; Bandler, J.W.; Madsen, Kaj
}

Published in:

IEEE Transactions on Microwave Theory and Techniques

Link to article, DOI:

10.1109/TMTT.2008.2011243

Publication date:

2009

Document Version

Publisher's PDF, also known as Version of record

Link back to DTU Orbit

Citation $(A P A)$ :

Koziel, S., Bandler, J. W., \& Madsen, K. (2009). Space Mapping With Adaptive Response Correction for Microwave Design Optimization. IEEE Transactions on Microwave Theory and Techniques, 57(2), 478-486. https://doi.org/10.1109/TMTT.2008.2011243

\section{General rights}

Copyright and moral rights for the publications made accessible in the public portal are retained by the authors and/or other copyright owners and it is a condition of accessing publications that users recognise and abide by the legal requirements associated with these rights.

- Users may download and print one copy of any publication from the public portal for the purpose of private study or research.

- You may not further distribute the material or use it for any profit-making activity or commercial gain

- You may freely distribute the URL identifying the publication in the public portal 


\title{
Space Mapping With Adaptive Response Correction for Microwave Design Optimization
}

\author{
Slawomir Koziel, Senior Member, IEEE, John W. Bandler, Life Fellow, IEEE, and Kaj Madsen
}

\begin{abstract}
Output space mapping is a technique introduced to enhance the robustness of the space-mapping optimization process in case the space-mapped coarse model cannot provide sufficient matching with the fine model. The technique often works very well; however, in some cases it fails. Especially in the microwave area where the typical model response (e.g., $\left|S_{21}\right|$ ) is a highly nonlinear function of the free parameter (e.g., frequency), the output spacemapping correction term may actually increase the mismatch between the surrogate and fine models for points other than the one at which the term was calculated, as in the surrogate model optimization process. In this paper, an adaptive response correction scheme is presented to work in conjunction with space-mapping optimization algorithms. This technique is designed to alleviate the difficulties of the standard output space mapping by adaptive adjustment of the response correction term according to the changes of the space-mapped coarse model response. Examples indicate the robustness of our approach.
\end{abstract}

Index Terms-Engineering optimization, microwave design, response correction, space mapping, space-mapping optimization.

\section{INTRODUCTION}

$\mathbf{S}$ PACE mapping has been used for solving difficult optimization problems in the microwave area for more than a decade [1]-[4]. This methodology is founded on the idea of the optimization of expensive or "fine" models by means of the iterative optimization and updating of so-called "coarse" models which are less accurate, but cheaper to evaluate. Provided that the misalignment between the fine and coarse model is not significant, space-mapping-based algorithms typically provide excellent results after only a few evaluations of the fine model. A similar idea is shared by other surrogate-model-based methods [5]-[11]; however, many of them do not use a simplified physically based coarse model: a functional surrogate is created by direct approximation of the available fine model data.

Space mapping is widely used in the optimization of microwave devices [1]-[3], [12]-[16], where fine models are

Manuscript received October 31, 2007; revised May 25, 2008. This work was supported in part by the Natural Sciences and Engineering Research Council of Canada (NSERC) under Grant RGPIN7239-06 and Grant STPGP336760-06, and by Bandler Corporation.

S. Koziel is with the School of Science and Engineering, Reykjavík University, Reykjavík IS-103, Iceland (e-mail: koziel@ru.is).

J. W. Bandler is with the Simulation Optimization Systems Research Laboratory, Department of Electrical and Computer Engineering, McMaster University, Hamilton, ON, Canada L8S 4K1, and also with Bandler Corporation, Dundas, ON, Canada L9H 5E7 (e-mail: bandler@mcmaster.ca).

K. Madsen is with Informatics and Mathematical Modelling, Technical University of Denmark, DK-2800 Lyngby, Denmark (e-mail: km@imm.dtu.dk).

Color versions of one or more of the figures in this paper are available online at http://ieeexplore.ieee.org.

Digital Object Identifier 10.1109/TMTT.2008.2011243 often based on full-wave electromagnetic simulations, whereas coarse models may be physically based circuit models. Recently, space-mapping techniques have been applied to design problems in a growing number of areas (e.g., [17]-[19]).

A number of papers cover different aspects of space mapping, including the development of new algorithms [2], [3], [20], [21], space-mapping-based modeling [22]-[24], neuro-spacemapping [25]-[28], theoretical foundations [20], [29], [30], etc.

One of the recent developments in space mapping is the so-called output space mapping [29], [31] in which the space-mapping surrogate model is created by enhancing the coarse model using a correction term, typically additive or multiplicative, that allows us to obtain a perfect matching between the fine model and a surrogate at the current iteration point. If sensitivity information is employed, the correction term can be constructed in such a way that the surrogate model matches the fine model with respect to response and first-order derivatives. If equipped with a trust region method [32], the output space-mapping algorithm with the surrogate satisfying first-order consistency conditions can be shown to converge to a local optimum of the fine model [20], [33]. Different versions of output space mapping have been described in the literature, e.g., [4], [20], [29].

Typically, output space mapping improves the performance of the space-mapping algorithm when applied as an auxiliary mapping. If the range of the coarse model is substantially different from the range of the fine model, output space mapping is practically mandatory to make the space-mapping algorithm perform reasonably well.

Unfortunately, in some cases output space mapping does not work as expected. Especially in the microwave area, the typical model response, e.g., the $\left|S_{21}\right|$ parameter, is a highly nonlinear function of the free parameter (typically the frequency of the input signal). In particular, the response may contain sharp minima corresponding to zeros of the transfer function of the underlying device. In such cases, the output space-mapping correction term may actually increase the apparent mismatch between the surrogate and fine model for points other than the one at which the term was calculated, i.e., during the process of surrogate model optimization. It may also severely deform the surrogate model response (see Section II for examples). As a consequence, the performance of the space-mapping algorithm may be degraded both with respect to the quality of the final solution as well as the computational cost of the optimization process.

In this paper, we propose an adaptive response correction scheme, which is designed to alleviate the difficulties of the standard output space mapping and improve the overall performance of the output space mapping. Our technique is based 
on the adaptive adjustment of the response correction term. The adjustment is related to the changes of the space-mapped coarse model response, which occury during the optimization of the surrogate. More specifically, the model response will be normally shifted, "squeezed" and/or "expanded" in frequency during the optimization process where we adjust the design parameters in order to have the model meet the design specifications. The output space-mapping correction term is kept constant (i.e., independent of the design variables) during the surrogate model optimization according to the standard approach. According to the adaptive response correction approach the correction term is being modified to make it change in frequency in the same way as the space-mapped coarse model response is changing. This technique is based on the assumption that the frequency responses of both the fine and space-mapped coarse model responses are modified in a similar way when the respective models are subjected to the same modification of the design parameters.

The robustness of the adaptive response correction method is demonstrated using microwave design optimization problems.

\section{ADAPTIVE RESPONSE CORRECTION}

\section{A. Motivation}

Let $\boldsymbol{R}_{f}: X_{f} \rightarrow R^{m}, X_{f} \subseteq R^{n}$, and $\boldsymbol{R}_{c}: X_{c} \rightarrow R^{m}, X_{c} \subseteq$ $R^{n}$ denote the response vectors of the fine and coarse models of a given microwave device, respectively. $R$ denotes the set of real numbers. Components of $\boldsymbol{R}_{f}$ and $\boldsymbol{R}_{c}$ may be evaluations of $\left|S_{21}\right|$ parameter at $m$ different frequencies. In particular, we may have $\boldsymbol{R}_{f}(\boldsymbol{x})=\left[R_{f}\left(\boldsymbol{x}, \omega_{1}\right) R_{f}\left(\boldsymbol{x}, \omega_{2}\right) \ldots R_{f}\left(\boldsymbol{x}, \omega_{m}\right)\right]^{T}$, where $R_{f}\left(\boldsymbol{x}, \omega_{j}\right)$ is a component of $\boldsymbol{R}_{f}$ corresponding to frequency $\omega_{j} ; \boldsymbol{x}$ denotes the vector of design parameters of the device.

The space-mapping optimization algorithm produces a sequence of points $\boldsymbol{x}^{(i)}, i=0,1,2, \ldots$, where $\boldsymbol{x}^{(i)}$ represents values of the design parameters (also called design variable vector) at iteration $i ; \boldsymbol{x}^{(i+1)}$ is an optimal solution (design) of the so-called surrogate model $\boldsymbol{R}_{s}^{(i)}$. Typically, $\boldsymbol{x}^{(0)}$ is the optimal solution of the coarse model. Surrogate model at iteration $i, \boldsymbol{R}_{s}^{(i)}$ is based on the coarse model and certain auxiliary mappings. Typically, output space mapping is used on the top of other space-mapping types. We shall use symbol $\overline{\boldsymbol{R}}_{c}^{(i)}$ to denote the space-mapped coarse model at iteration $i$, i.e., the coarse model composed with space mapping (or composition of more mappings if necessary; however, excluding the output space mapping that will be considered separately). For the sake of example, consider input space mapping, in which case $\overline{\boldsymbol{R}}_{c}^{(i)}(\boldsymbol{x})=\boldsymbol{R}_{c}\left(\boldsymbol{B}^{(i)} \cdot \boldsymbol{x}+\boldsymbol{c}^{(i)}\right)$, where $\boldsymbol{B}^{(i)}$ and $\boldsymbol{c}^{(i)}$ are mapping parameters obtained though parameter extraction [20]; $\boldsymbol{B}^{(i)}$ is an $n \times n$ matrix, while $\boldsymbol{c}^{(i)}$ is an $n \times 1$ vector.

The standard output space-mapping technique [29] assumes that the surrogate model $\boldsymbol{R}_{s}^{(i)}$ is defined as

$$
\boldsymbol{R}_{s}^{(i)}(\boldsymbol{x})=\overline{\boldsymbol{R}}_{c}^{(i)}(\boldsymbol{x})+\boldsymbol{d}^{(i)}
$$

with

$$
\boldsymbol{d}^{(i)}=\boldsymbol{R}_{f}\left(\boldsymbol{x}^{(i)}\right)-\overline{\boldsymbol{R}}_{c}^{(i)}\left(\boldsymbol{x}^{(i)}\right)
$$

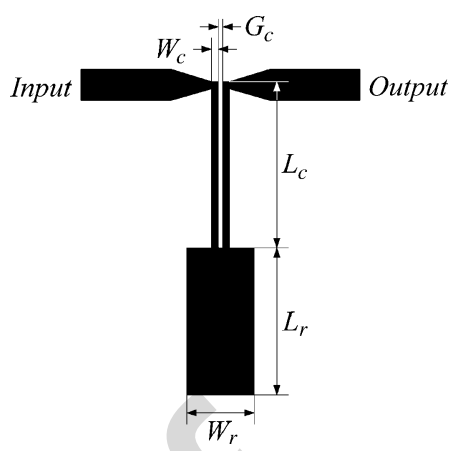

Fig. 1. Geometry of the wideband bandstop microstrip filter [34].

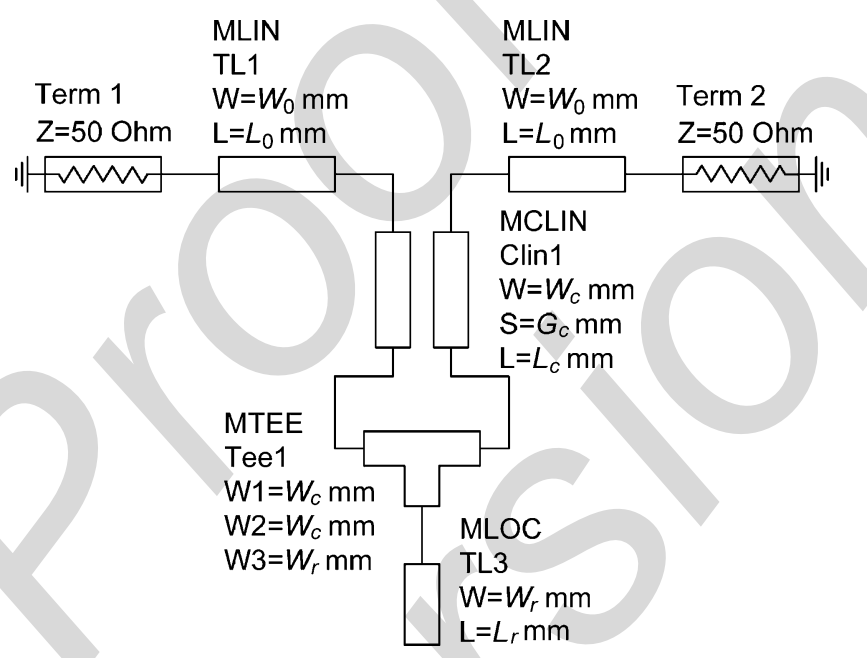

Fig. 2. Coarse model of the wideband bandstop filter (Agilent Technologies' ADS).

This definition ensures a zero-order consistency condition between the fine model and the space-mapping surrogate at $\boldsymbol{x}^{(i)}$, i.e., $\boldsymbol{R}_{f}\left(\boldsymbol{x}^{(i)}\right)=\boldsymbol{R}_{s}^{(i)}\left(\boldsymbol{x}^{(i)}\right)$.

Consider the following example: the wideband bandstop microstrip filter [34] shown in Fig. 1. The design parameters are $\boldsymbol{x}=\left[L_{r} W_{r} L_{c} W_{c} G_{c}\right]^{T}$. The fine model $\boldsymbol{R}_{f}$ is simulated in FEKO [35]. The codse model $\boldsymbol{R}_{c}$ is the circuit model implemented in Agnent Technologies' Advanced Design System (ADS) [36] (Fig. 2)

$$
\begin{aligned}
& \text { The design speci Put more spaces here } \\
& \left|S_{21}\right| \geq-3 \mathrm{~dB}, \quad \text { for } 1.0 \mathrm{GHz} \leq \omega \leq 2.0 \mathrm{GHz} \\
& \left|S_{21}\right| \leq-20 \mathrm{~dB}, \quad \text { for } 3.0 \mathrm{GHz} \leq \omega \leq 9.0 \mathrm{GHz} \\
& \left|S_{21}\right| \geq-3 \mathrm{~dB}, \quad \text { for } 10.0 \mathrm{GHz} \leq \omega \leq 11.0 \mathrm{GHz} .
\end{aligned}
$$

For this problem we use the input-space-mapped coarse model defined as $\bar{R}_{c}^{(i)}(\boldsymbol{x})=\boldsymbol{R}_{c}\left(\boldsymbol{x}+\boldsymbol{c}^{(i)}\right)$, where vector $\boldsymbol{c}^{(i)}$ is obtained using parameter extraction. On top of $\overline{\boldsymbol{R}}_{c}^{(i)}$, we use standard output space mapping, as defined in (1), (2).

Fig. 3 shows the fine and space-mapped coarse model responses at the starting point $\boldsymbol{x}^{(0)}=\left[\begin{array}{lllll}7.017 & 0.980 & 8.653 & 0.055 & 0.109\end{array}\right]^{T} \mathrm{~mm}$ (the coarse model optimal solution). Fig. 4 shows the response of the initial surrogate model $R_{s}^{(0)}$ at the starting point and at its optimal solution $\boldsymbol{x}^{(1)}$. We can observe that $\boldsymbol{R}_{s}^{(0)}\left(\boldsymbol{x}^{(1)}\right)$ is substantially distorted around $6 \mathrm{GHz}$, which is a consequence 


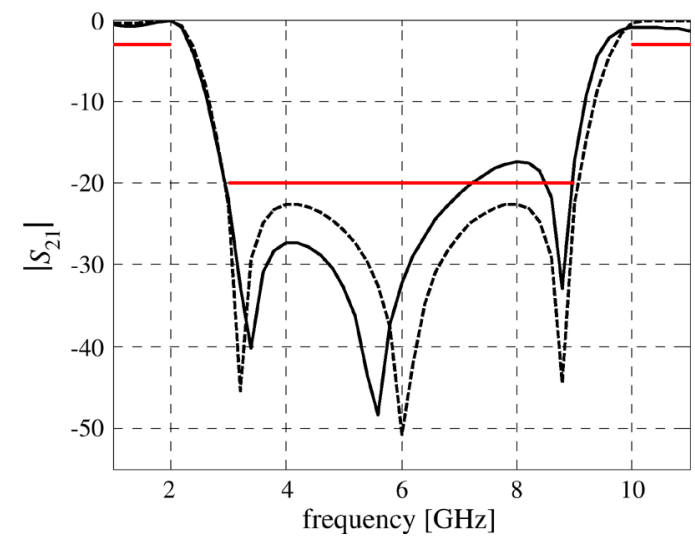

Fig. 3. Wideband bandstop filter: fine model (solid line) and space-mapped coarse model (dashed line) responses at $\boldsymbol{x}^{(0)}$.

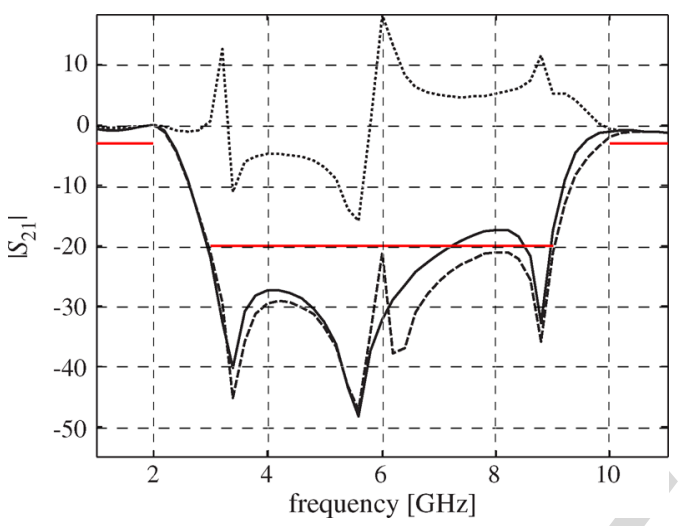

Fig. 4. Wideband bandstop filter: initial surrogate model response $\boldsymbol{R}_{\mathrm{s}}^{(0)}\left(\boldsymbol{x}^{(0)}\right)$ (solid line), optimized surrogate model response $\boldsymbol{R}_{s}^{(0)}\left(\boldsymbol{x}^{(1)}\right)$ (dashed line), and standard space-mapping correction term $\boldsymbol{d}^{(0)}$ (dotted line).

of the fact that the output space-mapping correction term $\boldsymbol{d}^{(0)}$ (shown in Fig. 4 as a dotted line) is a vector (independent of the design variables $\boldsymbol{x}$ ) with a sharp maximum around $6 \mathrm{GHz}$, while it is seen that the surrogate model optimization process tends to move the central zero of the filter into higher frequencies. In other words, the distortion due to the correction term $\boldsymbol{d}^{(0)}$ is a source of difficulty in the surrogate model optimization process.

The fine model specification error at $\boldsymbol{x}^{(0)}$ is $+2.7 \mathrm{~dB}$. Due to the above-mentioned problems, space-mapping optimization finds a solution with corresponding specification error $-1.1 \mathrm{~dB}$, which is rather poor as the minimax optimum for this problem is about $-2 \mathrm{~dB}$. The number of fine model evaluations required to find the solution (excluding evaluation at $\left.\boldsymbol{x}^{(0)}\right)$ is 8 .

\section{B. Adaptive Response Correction: Concept}

In order to avoid the problems highlighted above, we propose the following adaptive response correction technique. The surrogate model $\boldsymbol{R}_{s}^{(i)}$ at any iteration point $\boldsymbol{x}^{(i)}$ is defined as

$$
\boldsymbol{R}_{s}^{(i)}(\boldsymbol{x})=\overline{\boldsymbol{R}}_{c}^{(i)}(\boldsymbol{x})+\Delta_{r}\left(\boldsymbol{x}, \boldsymbol{x}^{(i)}\right)
$$

where $\boldsymbol{\Delta}_{r}\left(\boldsymbol{x}, \boldsymbol{x}^{(i)}\right)$ is the response correction term dependent on the design variables $x$. As we still want to maintain a perfect

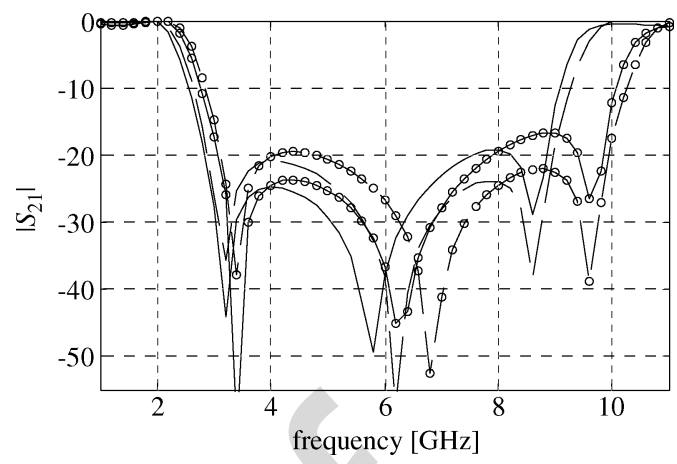

Fig. 5. Wideband bandstop filter: fine (solid line) and space-mapped coarse model (dashed line) responses at,certain point (design variable vector), as well as fine (solid line with circle markers) and space-mapped coarse model (dashed $a$ line with circle markers) responses at a different point.

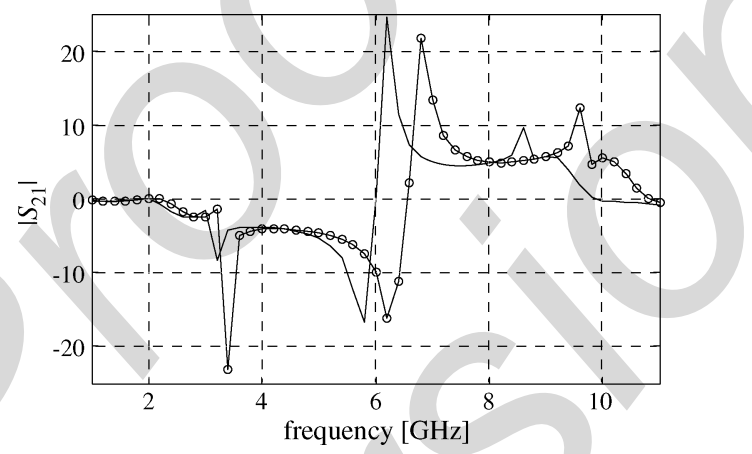

Fig. 6. Wideband bandstop filter: standard output space-mapping correction terms corresponding to responses shown in Fig. 5.

match between the fine model and the space-mapping surrogate at $\boldsymbol{x}^{(i)}, \boldsymbol{\Delta}_{r}\left(\boldsymbol{x}, \boldsymbol{x}^{(i)}\right)$ must satisfy

$$
\boldsymbol{\Delta}_{r}\left(\boldsymbol{x}^{(i)}, \boldsymbol{x}^{(i)}\right)=\boldsymbol{d}^{(i)}=\boldsymbol{R}_{f}\left(\boldsymbol{x}^{(i)}\right)-\overline{\boldsymbol{R}}_{c}^{(i)}\left(\boldsymbol{x}^{(i)}\right) .
$$

The idea behind the adaptive response correction is to account for the difference between the space-mapped coarse model response at $\boldsymbol{x}$ and at $\boldsymbol{x}^{(i)}$ and to modify the correction term accordingly with the initial correction $\boldsymbol{d}^{(i)}$ taken as a reference. In particular, we want to modify the initial correction term $\boldsymbol{d}^{(i)}$ in such a way that this modification reflects changes to $\overline{\boldsymbol{R}}_{c}^{(i)}$ during the process of surrogate model optimization. In particular. if the response of $\overline{\boldsymbol{R}}_{c}^{(i)}$ shifts or changes its shape with rAspect to frequency, the response correction term should track these changes.

As an illustration, consider Fig. 5, which shows the fine and space-mapped coarse model resnonses for the widehand handstop filter (Section II-A) at Remove 'In particular' hat the general relation between the mapped coarse model response and its corresponding fine model response is preserved (e.g., the center minimum of the mapped coarse model response is athigher frequency than the corresponding minimum for the a fine model). Fig. 6 shows the standard output space-mapping correction terms $\boldsymbol{d}$ corresponding to the responses shown in Fig. 5. As we can observe, the relation between these terms as functions of frequency is similar to the relation between the space-mapped coarse model responses so that proper tracking of coarse model changes help determine necessary changes to the output space-mapping corredtion terms. 
In our implementation, the tracking of the coarse model response changes is realized through a proper mapping that describes the shape relations between two coarse model responses and updates the response correction term so that the initial and current correction terms correspond to the same (frequencybased) relationship as the initial and current coarse model rePut more spaces here ription and technical details of this process are given below.

Let us assume without loss of generality that the components of $\boldsymbol{R}_{f}\left(\boldsymbol{R}_{c}\right)$ correspond to the model evaluations (e.g. $\aleph_{2} \downarrow$ at $m$ different frequency points, i.e., $\Omega=\left[\omega_{1} \omega_{2} \ldots \omega_{m}\right]^{T}$. For example, we have $\boldsymbol{R}_{f}(\boldsymbol{x})=$ $\left[R_{f}\left(\boldsymbol{x}, \omega_{1}\right) R_{f}\left(\boldsymbol{x}, \omega_{2}\right) \ldots R_{f}\left(\boldsymbol{x}, \omega_{m}\right)\right]^{T}$, where $R_{f}\left(\boldsymbol{x}, \omega_{j}\right)$ is a component of $\boldsymbol{R} f$ orresponding to frequency $\omega_{j}$. Let $I: R \times \Omega \times\left[\omega_{\min }, \omega_{\mathrm{max}}\right] \rightarrow R$ be an interpolation/extrapolation function such that $I(R, \Omega, \cdot)$ interpolates the response $R$ Put more spaces here $]$ and extrapolates $\boldsymbol{R}$ onto $\left[\omega_{\min }, \omega_{1}\right)$ and $\left(\omega_{m}, \omega_{\max }\right]$. In particular, $I(\boldsymbol{R}, \Omega, \omega)$ is the evaluation of the interpolated/extrapolated response $R$ at frequency $\omega$. In this paper, we use $I$ implemented as piecewise cubic splines (interpolation part) and linear extrapolation (extrapolation part).

The core of the adaptive response correction is a mapping $F^{(i)}: X_{q} \times\left[\omega_{1}, \omega_{m}\right] \rightarrow\left[\omega_{\min }, \omega_{\max }\right] . F^{(i)}$ is established at iteration $i$ in such a way that the difference between $\overline{\boldsymbol{R}}_{c}^{(i)}(\boldsymbol{x})$, i.e., the space-mapped coarse model response at $\boldsymbol{x}$ and $I\left(\overline{\boldsymbol{R}}_{c}^{(i)}\left(\boldsymbol{x}^{(i)}\right), \Omega, F^{(i)}(\boldsymbol{x}, \Omega)\right)$, i.e., $\overline{\boldsymbol{R}}_{c}^{(i)}\left(\boldsymbol{x}^{(i)}\right)$ interpolated/extrapolated through $I$ onto $\left[\omega_{\min }, \omega_{\max }\right]$ and evaluated at frequencies $F^{(i)}\left(\boldsymbol{x}, \omega^{(1)}, F^{(i)}\left(\boldsymbol{x}, \omega_{2}\right), \ldots, F^{(i)}\left(\boldsymbol{x}, \omega_{m}\right)\right.$ is minimized. Here, $X_{q}$ denotes the space-mapped coarse model domain. $F^{(i)}(\boldsymbol{x}, \Omega)$ will be referred to as mapped frequency.

In general, $\omega_{\min }\left(\omega_{\max }\right)$ may differ from $\omega_{1}\left(\omega_{m}\right)$, reParenthesis ')' is lacking here lel is not a constant Iuाction or Irequency arouाu $\omega_{1}\left(\omega_{m}\right)$, one may need to allow the mapped frequencies $n$ exceed the interval $\left[\omega_{1}, \omega_{m}\right]$ in order to make the good alignment between $\overline{\boldsymbol{R}}_{c}^{(i)}(\boldsymbol{x})$ and $I\left(\overline{\boldsymbol{R}}_{c}^{(i)}\left(\boldsymbol{x}^{(i)}\right), \Omega, F^{(i)}(\boldsymbol{x}, \Omega)\right)$ possible. For example, if $\overline{\boldsymbol{R}}_{c}^{(i)}(\boldsymbol{x})$ is larger than $\overline{\boldsymbol{R}}_{c}^{(i)}\left(\boldsymbol{x}^{(i)}\right)$ at $\omega_{1}$ and both are decreasing functions of frequency around $\omega_{1}$, the only way to make it possible to align $\overline{\boldsymbol{R}}_{c}^{(i)}(\boldsymbol{x})$ with $I\left(\overline{\boldsymbol{R}}_{c}^{(i)}\left(\boldsymbol{x}^{(i)}\right), \Omega, F^{(i)}(\boldsymbol{x}, \Omega)\right)$ at $\omega_{1}$ would be to allow $\omega_{\min }<\omega_{1}$ so that $F^{(i)}\left(\boldsymbol{x}, \omega_{1}\right)<\omega_{1}$ and $I\left(\overline{\boldsymbol{R}}_{c}^{(i)}\left(\boldsymbol{x}^{(i)}\right), \Omega, F^{(i)}(\boldsymbol{x}, \Omega)\right)$ at $\omega_{1}$ would refer to $\overline{\boldsymbol{R}}_{c}^{(i)}\left(\boldsymbol{x}^{(i)}\right)$ for frequencies smaller than $\omega_{1}$. In practice, we may want to set $\omega_{\min }$ to be slightly smaller than $\omega_{1}$ ( $\omega_{\max }$ to be slightly larger than $\omega_{m}$ ).

The vector $I\left(\overline{\boldsymbol{R}}_{c}^{(i)}\left(\boldsymbol{x}^{(i)}\right), \Omega, F^{(i)}(\boldsymbol{x}, \Omega)\right)$ is nothing but $\overline{\boldsymbol{R}}_{c}^{(i)}\left(\boldsymbol{x}^{(i)}\right)$ scaled with respect to frequency in order to be as similar to $\overline{\boldsymbol{R}}_{c}^{(i)}(\boldsymbol{x})$ as possible in a given sense. In other words, mapping $F^{(i)}$ is supposed to be defined in such a way that mapped frequency $F^{(i)}(x, \Omega)$ reflects the shape change of the space-mapped coarse model response at $\boldsymbol{x}$ with respect to its original shape at $\boldsymbol{x}^{(i)}$. For the sake of consistency with condition (4), $F^{(i)}\left(x^{(i)}, \Omega\right)$ should be equal $\Omega$, i.e., the mapped frequency should be the same as the original frequency at $\boldsymbol{x}^{(i)}$.
Having the mappings $I$ and $F^{(i)}$, we can define the response correction term as follows:

$\boldsymbol{\Delta}_{r}\left(\boldsymbol{x}, \boldsymbol{x}^{(i)}\right)=I\left(\boldsymbol{R}_{f}\left(\boldsymbol{x}^{(i)}\right)-\overline{\boldsymbol{R}}_{c}^{(i)}\left(\boldsymbol{x}^{(i)}\right), \Omega, F^{(i)}(\boldsymbol{x}, \Omega)\right)_{(5)}$

The reason for using function $I$ is the fact that the entire process requires information about $\overline{\boldsymbol{R}}_{c}^{(i)}\left(\boldsymbol{x}^{(i)}\right)$ and $\boldsymbol{R}_{f}\left(\boldsymbol{x}^{(i)}\right)-$ $\overline{\boldsymbol{R}}_{c}^{(i)}\left(\boldsymbol{x}^{(i)}\right)$ at frequencies different from the original evaluation frequencies $\omega_{1}, \omega_{2}, \ldots, \omega_{m}$.

In the following sections, we describe two realizations of the mapping $F^{(i)}$. Other possible realizations will be dealt with elsewhere.

\section{Mapping F Implementation: Realization 1}

This realization is suitable for problems in which the coarse model response is rather smooth as a function of frequency (i.e., without sharp local minima and maxima) and such that the range of the response (as a function of frequency) does not change much with $\boldsymbol{x}$.

Put more spaces here,$\omega)$ is defined as

$$
F^{(i)}(\boldsymbol{x}, \psi)=\left\lceil\boldsymbol{\lambda}^{(i)}(\boldsymbol{x})\right]^{T} \boldsymbol{v}(\omega)
$$

where $\boldsymbol{\lambda}^{(i)}(\boldsymbol{x})=\left[\lambda_{1}^{(i)}(\boldsymbol{x}) \lambda_{2}^{(i)}(\boldsymbol{x}) \ldots \lambda_{p}^{(i)}(\boldsymbol{x})\right]^{T}$ are scaling coefficient $\sqrt{\text { Put more spaces here }}$

$\boldsymbol{\lambda}^{(i)}(\boldsymbol{x})=\arg \min _{\boldsymbol{\lambda}}\left\|I\left(\overline{\boldsymbol{R}}_{\oint}^{(i)}\left(\boldsymbol{x}^{(i)}\right), \Omega, \boldsymbol{\lambda}^{T} \boldsymbol{v}(\Omega)\right)-\overline{\boldsymbol{R}}_{c}^{(i)}(\boldsymbol{x})\right\|$ while $\boldsymbol{v}(\omega)=\left[v_{1}(\omega) v_{2}(\omega) \ldots v_{p}(\omega)\right]^{T}$ are basis functions. Optimization problem (6), which is readily solved by MATLAB, has the following constraints: a) $d\left(\left[\boldsymbol{\lambda}^{(i)}(\boldsymbol{x})\right]^{T} \boldsymbol{v}(\omega)\right) / d \omega>0$, i.e., scaling function $F$ is monotonic; b) $\left[\boldsymbol{\lambda}^{(i)}(\boldsymbol{x})\right]^{T} \boldsymbol{v}\left(\omega_{1}\right) \geq \omega_{\min }$; and c) $\left[\lambda^{(i)}(\boldsymbol{x})\right]^{T} \boldsymbol{v}\left(\omega_{m}\right) \leq \omega_{\max }$, i.e., we need to ensure that $F^{(i)}(\boldsymbol{x}, \omega)$ is a monotonic function of $\omega$ within its range in $\left[\omega_{\min }, \omega_{\max }\right]$. In this paper, we use a third-order polynomial scaling, i.e., we have $p=4$ and $\boldsymbol{v}(\omega)=\left[1 \omega \omega^{2} \omega^{3}\right]^{T}$. Typically $\omega_{\min }$ is slightly smaller than $\omega_{1}$ (e.g., b $10 \%-20 \%$ ), and $\omega_{\max }$ is slightly larger than $\omega_{m}$.

\section{Mapping F Implementation: Re}

Put more spaces here

This realization is suitable for problems in which the coarse model response contains clearly defined characteristic frequencies, e.g., sharp minima corresponding to zeros of the transfer function.

Let $\Omega_{1}=\left\{\omega_{1.1}, \omega_{1.2}, \ldots, \omega_{1 . N}\right\}$ and $\Omega_{2}=$ $\left\{\omega_{2.1}, \omega_{2.2}, \ldots, \omega_{2 . N}\right\}$ be the sets of characteristic frequencies of $\overline{\boldsymbol{R}}_{c}^{(i)}\left(\boldsymbol{x}^{(i)}\right)$ and $\overline{\boldsymbol{R}}_{c}^{(i)}(\boldsymbol{x})$, respectively. The mapped frequency $F^{(i)}(\boldsymbol{x}, \omega)$ is defined as an interpolation function such that $F^{(i)}\left(\boldsymbol{x}, \omega_{2 . j}\right)=\omega_{1 . j}, j=1,2, \ldots, N$. In this paper, we implement $F$ using cubic splines. If there is a different number of characteristic frequencies for $\overline{\boldsymbol{R}}_{c}^{(i)}(\boldsymbol{x})$ and $\overline{\boldsymbol{R}}_{c}^{(i)}\left(\boldsymbol{x}^{(i)}\right)$, both $\Omega_{1}$ and $\Omega_{2}$ are adjusted using an auxiliary algorithm in order to maintain the correspondence between the two sets. In order to have the mapped frequency $F^{(i)}(\boldsymbol{x}, \omega)$ well defined on $\left[\omega_{1}, \omega_{m}\right]$, we typically set $\omega_{1.1}=\omega_{2.1}=\omega_{1}$ and $\omega_{1 . N}=\omega_{2 . N}=\omega_{m}$. However, if there is a difference between 
TABLE I

SPACE-MAPPING OPTIMIZATION RESUlTS FOR THE WIDEBAND BANDSTOP MICROSTRIP FILTER

\begin{tabular}{ccc}
\hline \hline Output Space Mapping Used & $\begin{array}{c}\text { Final Spec. Error } \\
{[\mathrm{dB}]}\end{array}$ & $\begin{array}{c}\text { Number of Fine Model } \\
\text { Evaluations }\end{array}$ \\
\hline Standard Output Space Mapping & -1.1 & 8 \\
Adaptive Response Correction & -2.1 & 3 \\
\hline \hline
\end{tabular}

* Excludes the fine model evaluation at the starting point.

$\overline{\boldsymbol{R}}_{c}^{(i)}(\boldsymbol{x})$ and $\overline{\boldsymbol{R}}_{c}^{(i)}\left(\boldsymbol{x}^{(i)}\right)$ at $\omega_{1}$, then it is better to set $\omega_{1.1}$ to $\bar{\omega}=\arg \min _{\omega}\left|I\left(\overline{\boldsymbol{R}}_{c}^{(i)}\left(\boldsymbol{x}^{(i)}\right), \Omega, \omega\right)-\overline{\boldsymbol{R}}_{c}^{(i)}\left(\boldsymbol{x}, \omega_{1}\right)\right|$ in order to obId $R$ tain a better overall match between $I\left(\overline{\boldsymbol{R}}_{c}^{(i)}\left(\boldsymbol{x}^{(i)}\right), \Omega, F^{(i)}(\boldsymbol{x}, \Omega)\right)$ and $\overline{\boldsymbol{R}}_{c}^{(i)}(\boldsymbol{x})$. A similar adjustment can be done for $\omega_{1 . N}$.

In addition to frequency scaling, there is the possibility of scaling the response correction term with respect to its amplitude, also based on the response change of the space-mapped coarse model. In this paper, however, we do not use this approach.

It should be noted that, although we assumed that the model response components are related through a free parameter, such as frequency, the adaptive response correction technique should work even if such a relation is not explicitly stated. One can consider the response component indices as values of the "free" parameter.

\section{EXAMPLES}

\section{A. Wideband Bandstop Filter}

As a first example, we again consider the wideband bandstop filter described in Section II. We optimized this filter using the input-space-mapped coarse model $\overline{\boldsymbol{R}}_{c}^{(i)}(\boldsymbol{x})=\boldsymbol{R}_{c}\left(\boldsymbol{x}+\boldsymbol{c}^{(i)}\right)$ and the adaptive response correction technique of Section II. We used Realization 2 of the scaling function $F$ as the filter response contains three clearly defined characteristic points. Table I shows the optimization results, as well as a comparison with the standard output space mapping. Note that the quality of solution found with the adaptive response correction is better than the one obtained with the standard method. The number of fine model evaluations required to find the solution is also substantially smaller for the adaptive correction technique.

In order to understand the performance of the adaptive response correction technique, consider Fig. 7 showing the response of initial surrogate model $\boldsymbol{R}_{s}^{(0)}$ at the starting point and at its optimal solution $\boldsymbol{x}^{(1)}$. We can observe that, in contrast to the standard output space mapping (cf. Fig. 4), $\boldsymbol{R}_{s}^{(0)}\left(\boldsymbol{x}^{(1)}\right)$ is not significantly distorted, which is because the response correction term is modified to reflect the change of $\overline{\boldsymbol{R}}_{c}^{(i)}\left(\boldsymbol{x}^{(1)}\right)$ with respect to $\overline{\boldsymbol{R}}_{c}^{(i)}\left(\boldsymbol{x}^{(0)}\right)$. Fig. 8 shows the correction terms $\Delta_{r}\left(x^{(0)}, \boldsymbol{x}^{(0)}\right)=\boldsymbol{d}^{(0)}$ and $\boldsymbol{\Delta}_{r}\left(\boldsymbol{x}^{(1)}, \boldsymbol{x}^{(0)}\right)$. Fig. 9 shows the plot of the scaling function $F^{(0)}\left(\boldsymbol{x}^{(1)}, \omega\right)$. The fine model response at the design found by the space-mapping algorithm with the adaptive response correction is shown in Fig. 10.

\section{B. Capacitively Coupled Dual-Behavior Resonator Filter}

Our second example is a second-order capacitively coupled dual-behavior resonator microstrip filter [37] shown in Fig. 11.

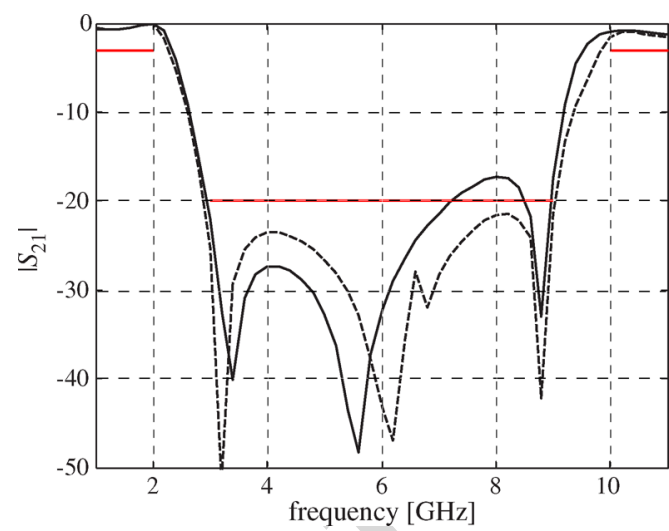

Fig. 7. Wideband bandstop filter: surrogate model response $\boldsymbol{R}_{s}^{(0)}\left(\boldsymbol{x}^{(0)}\right)$ (solid line) and optimized surrogate model response $\boldsymbol{R}_{s}^{(0)}\left(\boldsymbol{x}^{(1)}\right)$ (dashed line). Plots obtained for the adaptive response correction method.

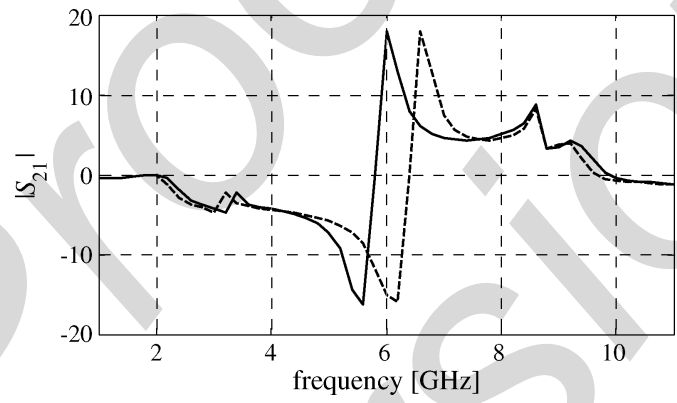

Fig. 8. Wideband bandstop filter: correction terms $\Delta_{r}\left(\boldsymbol{x}^{(0)}, \boldsymbol{x}^{(0)}\right)$ (solid line), and $\Delta_{r}\left(\boldsymbol{x}^{(1)}, \boldsymbol{x}^{(0)}\right)$ (dashed line). Plots obtained for the adaptive response correction method.

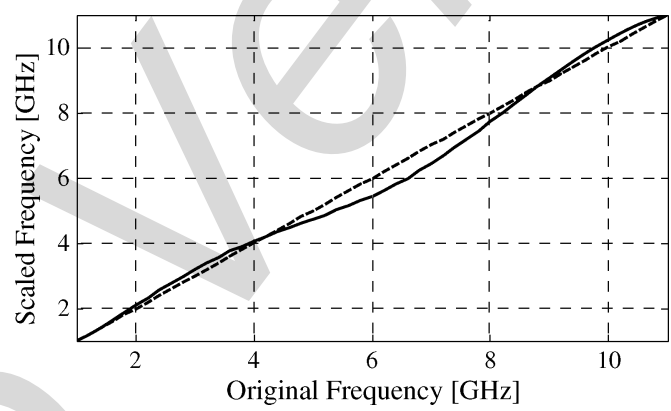

Fig. 9. Wideband bandstop filter: scaling function $F^{(0)}\left(\boldsymbol{x}^{(1)}, \omega\right)$ (solid line). Plot obtained for the adaptive response correction method. The identity function plot is shown as fashed line.

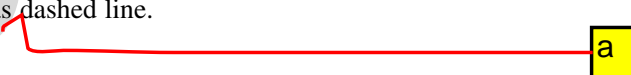

The design parameters are $\boldsymbol{x}=\left[\begin{array}{llll}L_{1} & L_{2} & L_{3} & S\end{array}\right]^{T}$. The fine model $\boldsymbol{R}_{f}$ is simulated in FEKO [35]. The coarse model $\boldsymbol{R}_{c}$ is the circuit model implemented in Agilent Technologies' ADS [36] and shown in Fig. 12. The design specifications for the filter are

$$
\begin{aligned}
& \left|S_{21}\right| \leq-20 \mathrm{~dB}, \quad \text { for } 2.0 \mathrm{GHz} \leq \omega \leq 3.2 \mathrm{GHz} \\
& \left|S_{21}\right| \geq-3 \mathrm{~dB}, \quad \text { for } 3.8 \mathrm{GHz} \leq \omega \leq 4.2 \mathrm{GHz} \\
& \left|S_{21}\right| \leq-20 \mathrm{~dB}, \quad \text { for } 4.8 \mathrm{GHz} \leq \omega \leq 6.0 \mathrm{GHz} .
\end{aligned}
$$

For this problem, we use the input-space-mapped coarse model $\overline{\boldsymbol{R}}_{c}^{(i)}(\boldsymbol{x})=\boldsymbol{R}_{c}\left(\boldsymbol{x}+\boldsymbol{c}^{(i)}\right)$ enhanced by a frequency space mapping in which the coarse model is evaluated at a frequency different than the fine model according to the affine mapping 


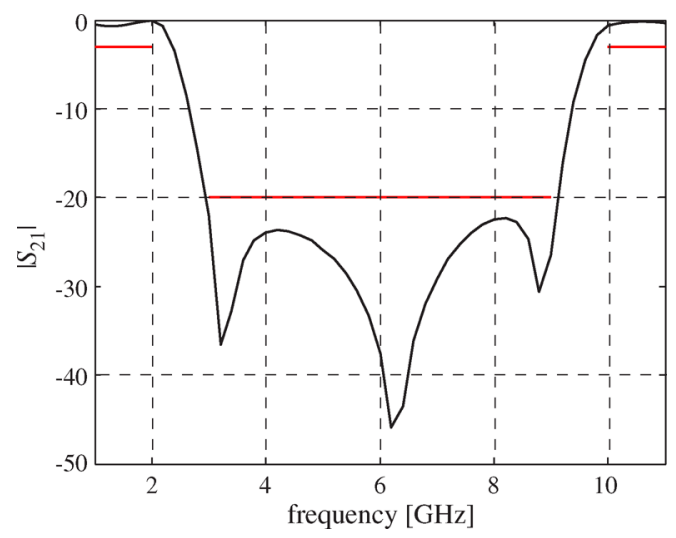

Fig. 10. Wideband bandstop filter: fine model response at the design found by the space-mapping optimization algorithm with the adaptive response correction (Realization 2).

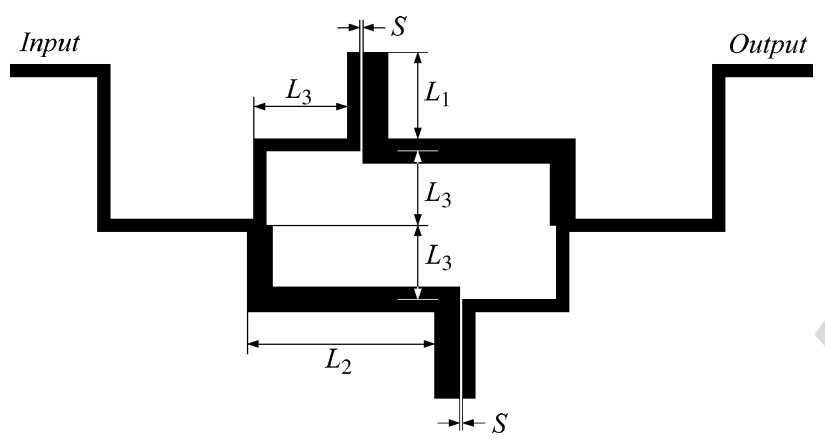

Fig. 11. Geometry of the capacitively coupled dual-behavior resonator microstrip filter [37].

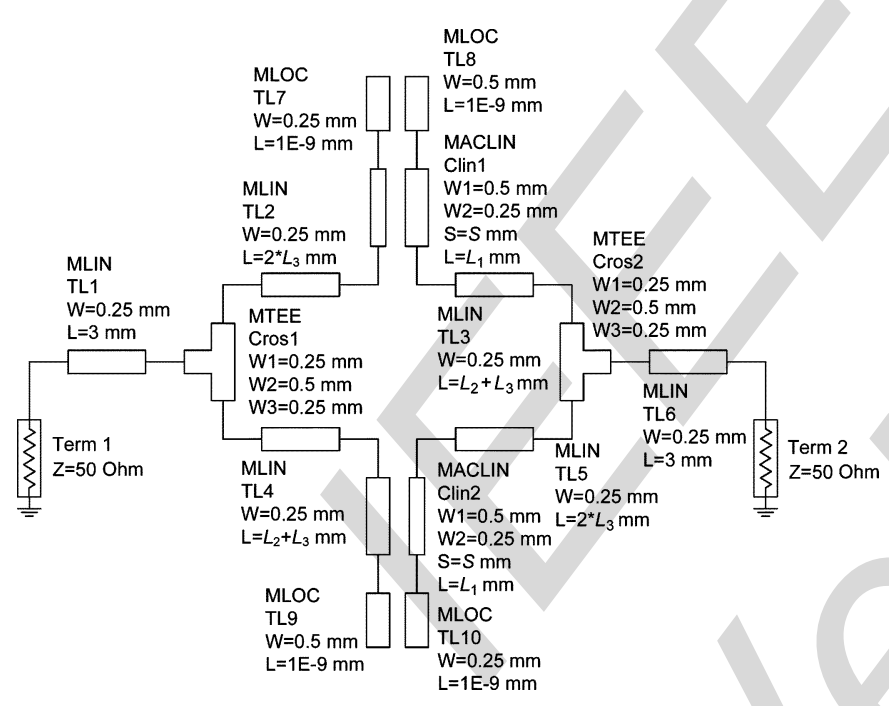

Fig. 12. Coarse model of the capacitively coupled dual-behavior resonator microstrip filter (Agilent Technologies' Put more spaces here

$\omega \leftarrow f_{1}+f_{2} \omega$. Both $\boldsymbol{c}^{(i)}$ and $\boldsymbol{F}^{(i)}=\left[f_{1} f_{2}\right]$ are found at iteration $i$ using parameter extraction. The starting point is the coarse model optimal solution $\boldsymbol{x}^{(0)}=[2.4156 .0931 .1670 .082]^{T} \mathrm{~mm}$. Fig. 13 shows the coarse and fine model responses at $\boldsymbol{x}^{(0)}$. The fine model specification error at $\boldsymbol{x}^{(0)}$ equals $+7.8 \mathrm{~dB}$.

Space-mapping optimization was performed three times using the space-mapped coarse model $\overline{\boldsymbol{R}}_{c}^{(i)}$ with: 1) the standard output space mapping (1), (2); 2) Realization 1 of the

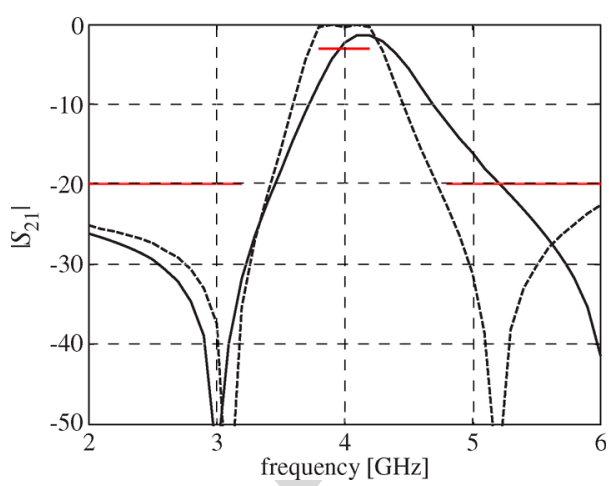

Fig. 13. Capacitively coupled dual-behavior resonator microstrip filter: coarse (dashed line) and fine (solid line) model response at the starting point $\boldsymbol{x}^{(0)}$.

TABLE II

SPACE-MAPPING OPTIMIZATION RESULTS FOR THE CAPACITIVELY COUPLED MICROSTRIP FILTER

\begin{tabular}{ccc}
\hline \hline Output Space Mapping Used & $\begin{array}{c}\text { Final Spec. Error } \\
{[\mathrm{dB}]}\end{array}$ & $\begin{array}{c}\text { Number of Fine Model } \\
\text { Evaluations }\end{array}$ \\
\hline Standard Output Space Mapping & -1.9 & 8 \\
Adaptive Response Correction & -2.5 & 5 \\
(Realization 1) & $(-2.1)$ & $(3)$ \\
Adaptive Response Correction & -2.0 & 3 \\
(Realization 2) & & \\
\hline \hline
\end{tabular}

* Excludes the fine model evaluation at the starting point.

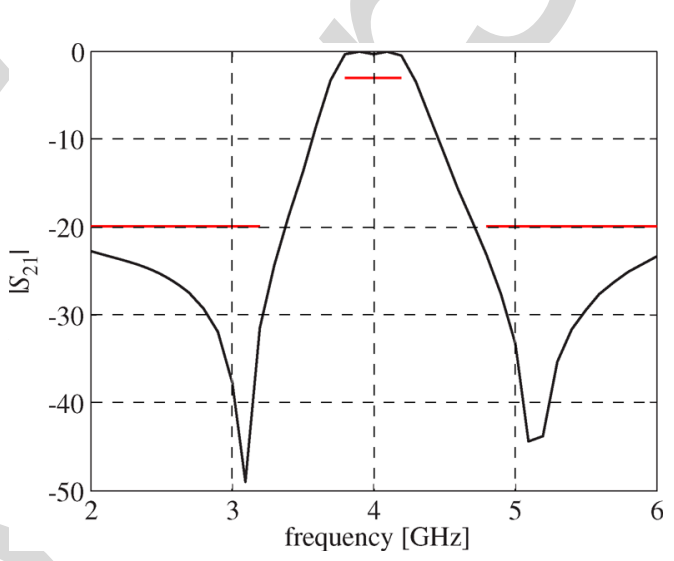

Fig. 14. Capacitively coupled dual-behavior resonator microstrip filter: fine model response at the design found by the space-mapping optimization algorithm with the adaptive response correction (Realization 1).

adaptive response correction technique of Section II; and 3) Realization 2 of the adaptive response correction method.

Table II shows the optimization results for the three methods. The quality of the solution found with the adaptive response correction (both for Realization 1 and Realization 2) is better than the quality of the solution obtained with the standard output space mapping. The number of fine model evaluations required to find the solution is smaller for the adaptive correction technique. It also follows from the results shown in Table II that Realization 1 of the adaptive response correction is slightly more suitable for our filter than Realization 2. Fig. 14 shows the fine model response at the final solution found by the space mapping with Realization 1 of the adaptive response correction. 
occur while optimizing the space-mapping surrogate model. This allows us to alleviate the difficulties of the standard output space mapping that uses a design-variable-independent correction term, which may result in serious distortion of the surrogate model response, and, consequently, deterioration of the performance of the space-mapping algorithm. The robustness of our technique is demonstrated using several microwave design optimization problems.

\section{ACKNOWLEDGMENT}

The authors thank Sonnet Software Inc., Syracuse, NY, for $\boldsymbol{e m}$ and Agilent Technologies, Santa Rosa, CA, for making ADS available.

\section{REFERENCES}

[1] J. W. Bandler, R. M. Biernacki, S. H. Chen, P. A. Grobelny, and R. H. Hemmers, "Space mapping technique for electromagnetic optimization," IEEE Trans. Microw. Theory Tech., vol. 4, no. 12, pp. 536-544, Dec. 1994.

[2] J. W. Bandler, Q. S. Cheng, N. K. Nikolova, and M. A. Ismail, "Implicit space mapping optimization exploiting preassigned parameters," IEEE Trans. Microw. Theory Tech., vol. 52, no. 1, pp. 378-385, Jan. 2004.

[3] J. W. Bandler, Q. S. Cheng, S. A. Dakroury, A. S. Mohamed, M. H. Bakr, K. Madsen, and J. Sondergaard, "Space mapping: The state of the art," IEEE Trans. Microw. Theory Tech., vol. 52, no. 1, pp. 337-361, Jan. 2004.

[4] D. Echeverria and P. W. Hemker, "Space mapping and defect correction," Int. Math. J. Comput. Methods Appl. Math., vol. 5, no. 2, pp. 107-136, 2005.

[5] N. M. Alexandrov and R. M. Lewis, "An overview of first-order model management for engineering optimization," Optim. Eng., vol. 2, no. 4, pp. 413-430, Dec. 2001.

[6] A. J. Booker, J. E. Dennis, Jr., P. D. Frank, D. B. Serafini, V. Torczon, and M. W. Trosset, "A rigorous framework for optimization of expensive functions by surrogates," Struct. Optim., vol. 17, no. 1, pp. 1-13, Feb. 1999.

[7] J. E. Dennis and V. Torczon, "Managing approximation models in optimization," in Multidisciplinary Design Optimization, N. M. Alexandrov and M. Y. Hussaini, Eds. Philadelphia, PA: SIAM, 1997, pp. 330-374.

[8] S. J. Leary, A. Bhaskar, and A. J. Keane, “A knowledge-based approach to response surface modeling in multifidelity optimization," Global Optim., vol. 26, no. 3, pp. 297-319, Jul. 2003.

[9] S. E. Gano, J. E. Renaud, and B. Sanders, "Variable fidelity optimization using a kriging based scaling function," in Proc. 10th AIAA/ISSMO Multidisciplinary Anal. Optim. Conf., Albany, NY, 2004.

[10] T. W. Simpson, J. Peplinski, P. N. Koch, and J. K. Allen, "Metamodels for computer-based engineering design: Survey and recommendations," Eng. Comput., vol. 17, no. 2, pp. 129-150, Jul. 2001.

[11] N. V. Queipo, R. T. Haftka, W. Shyy, T. Goel, R. Vaidynathan, and P. K. Tucker, "Surrogate-based analysis and optimization," Progr. Aerosp. Sci., vol. 41, no. 1, pp. 1-28, Jan. 2005.

[12] M. A. Ismail, D. Smith, A. Panariello, Y. Wang, and M. Yu, "EMbased design of large-scale dielectric-resonator filters and multiplexers by space mapping," IEEE Trans. Microw. Theory Tech., vol. 52, no. 1, pp. 386-392, Jan. 2004.

[13] K.-L. Wu, Y.-J. Zhao, J. Wang, and M. K. K. Cheng, "An effective dynamic coarse model for optimization design of LTCC RF circuits with aggressive space mapping," IEEE Trans. Microw. Theory Tech., vol. 52, no. 1, pp. 393-402, Jan. 2004.

[14] S. Amari, C. LeDrew, and W. Menzel, "Space-mapping optimization of planar coupled-resonator microwave filters," IEEE Trans. Microw. Theory Tech., vol. 54, no. 5, pp. 2153-2159, May 2006.

[15] M. Dorica and D. D. Giannacopoulos, "Response surface space mapping for electromagnetic optimization," IEEE Trans. Magn., vol. 42, no. 4, pp. 1123-1126, Apr. 2006.

[16] J. E. Rayas-Sánchez and V. Gutiérrez-Ayala, "EM-based Monte Carlo analysis and yield prediction of microwave circuits using linear-input neural-output space mapping," IEEE Trans. Microw. Theory Tech., vol. 54, no. 12, pp. 4528-4537, Dec. 2006.
[17] S. J. Leary, A. Bhaskar, and A. J. Keane, "A constraint mapping approach to the structural optimization of an expensive model using surrogates," Optim. Eng., vol. 2, no. 4, pp. 385-398, Dec. 2001.

[18] M. Redhe and L. Nilsson, "Using space mapping and surrogate models to optimize vehicle crashworthiness design," presented at the 9th AIAA/ISSMO Multidisciplinary Anal. Optim. Symp., Atlanta, GA, Sep. 2002, Paper AIAA-2002-5536.

[19] H.-S. Choi, D. H. Kim, I. H. Park, and S. Y. Hahn, "A new design technique of magnetic systems using space mapping algorithm," IEEE Trans. Magn., vol. 37, no. 5, pp. 3627-3630, Sep. 2001.

[20] S. Koziel, J. W. Bandler, and K. Madsen, "A space mapping framework for engineering optimization: Theory and implementation," IEEE Trans. Microw. Theory Tech., vol. 54, no. 10, pp. 3721-3730, Oct. 2006.

[21] S. Koziel and J. W. Bandler, "Space-mapping optimization with adaptive surrogate model," IEEE Trans. Microw. Theory Tech., vol. 55, no. 3, pp. 541-547, Mar. 2007.

[22] S. Koziel, J. W. Bandler, A. S. Mohamed, and K. Madsen, "Enhanced surrogate models for statistical design exploiting space mapping technology," in IEEE MTT-S Int. Microw. Symp. Dig., Long Beach, CA, Jun. 2005, pp. 1609-1612.

[23] S. Koziel, J. W. Bandler, and K. Madsen, "Theoretical justification of space-mapping-based modeling utilizing a data base and on-demand parameter extraction," IEEE Trans. Microw. Theory Tech., vol. 54, no. 12, pp. 4316-4322, Dec. 2006.

[24] S. Koziel and J. W. Bandler, "Microwave device modeling using space-mapping and radial basis functions," in IEEE MTT-S Int. Microw. Symp. Dig., Honolulu, HI, 2007, pp. 799-802.

[25] V. K. Devabhaktuni, B. Chattaraj, M. C. E. Yagoub, and Q.-J. Zhang, "Advanced microwave modeling framework exploiting automatic model generation, knowledge neural networks, and space mapping," IEEE Trans. Microw. Theory Tech., vol. 51, no. 7, pp. 1822-1833, Jul. 2003.

[26] J. E. Rayas-Sánchez, "EM-based optimization of microwave circuits using artificial neural networks: The state-of-the-art," IEEE Trans. Microw. Theory Tech., vol. 52, no. 1, pp. 420-435, Jan. 2004.

[27] J. E. Rayas-Sánchez, F. Lara-Rojo, and E. Martinez-Guerrero, "A linear inverse space-mapping (LISM) algorithm to design linear and nonlinear RF and microwave circuits," IEEE Trans. Microw. Theory Tech., vol. 53, no. 3, pp. 960-968, Mar. 2005.

[28] L. Zhang, J. Xu, M. C. E. Yagoub, R. Ding, and Q.-J. Zhang, "Efficient analytical formulation and sensitivity analysis of neuro-space mapping for nonlinear microwave device modeling," IEEE Trans. Microw. Theory Tech., vol. 53, no. 9, pp. 2752-2767, Sep. 2005.

[29] S. Koziel, J. W. Bandler, and K. Madsen, "Towards a rigorous formulation of the space mapping technique for engineering design," in Proc. Int. Symp. Circuits, Syst., Kobe, Japan, May 2005, pp. 5605-5608.

[30] K. Madsen and J. Søndergaard, "Convergence of hybrid space mapping algorithms," Optim. Eng., vol. 5, no. 2, pp. 145-156, Jun. 2004.

[31] J. W. Bandler, Q. S. Cheng, D. H. Gebre-Mariam, K. Madsen, F. Pedersen, and J. Søndergaard, "EM-based surrogate modeling and design exploiting implicit, frequency and output space mappings," in IEEE MTT-S Int. Microw. Symp. Dig., Philadelphia, PA, Jun. 2003, pp. 1003-1006.

[32] A. R. Conn, N. I. M. Gould, and P. L. Toint, Trust Region Methods, ser. MPS-SIAM Optim. Philadelphia, PA: SIAM, 2000.

[33] N. M. Alexandrov, J. E. Dennis, R. M. Lewis, and V. Torczon, "A trustregion framework for managing the use of approximation models in optimization," Struct. Optim., vol. 15, no. 1, pp. 16-23, 1998.

[34] M. Y. Hsieh and S. M. Wang, "Compact and wideband microstrip bandstop filter," IEEE Microw. Wireless Compon. Lett., vol. 15, no. 7, pp. 472-474, Jul. 2005.

[35] "FEKO User's Manual Suite 5.3" EM Softw. Syst. S.A. (Pty) Ltd., Stellenbosch, South Africa, 2008. [Online]. Available: http://www.feko.info

[36] Agilent Advanced Design System (ADS). ver. 2008, Agilent Technol., Santa Rosa, CA, 2008.

[37] A. Manchec, C. Quendo, J.-F. Favennec, E. Rius, and C. Person, "Synthesis of capacitive-coupled dual-behavior resonator (CCDBR) filters," IEEE Trans. Microw. Theory Tech., vol. 54, no. 6, pp. 2346-2355, Jun. 2006.

[38] W. H. Tu and K. Chang, "Microstrip elliptic-function low-pass filters using distributed elements or slotted ground structure," IEEE Trans. Microw. Theory Tech., vol. 54, no. 10, pp. 3786-3792, Oct. 2006.

[39] em. ver. 11.53, Sonnet Softw. Inc., North Syracuse, NY, 2008. 
received the M.Sc. and Ph.D. degrees in electronic

engineering from Gdansk University of Technology, Poland, in 1995 and 2000, respectively. He also received the M.Sc.

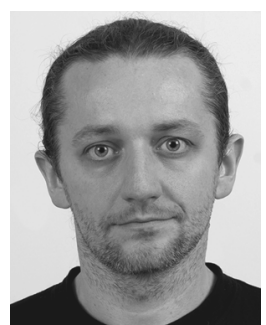

Slawomir Koziel (M'03-SM'07) fecelved the M.Sc. and Ph.D. degrees in electronic engineering M.Sc. degrees in theoretical physics and in mathematics, and Ph.D. degree im mathematics from Gdansk University of Technology, Gdansk Poland, in 1995, 2000, 2000, 2002, and 2003, respectively.

He was a Research Associate with the Department of Electrical and Computer Engineering, McMaster University, Hamilton, ON, Canada. He is currently with the School of Science and Engineering, Reykjavík University, Reykjavík, Iceland. He has authored or coauthored over 120 papers. His research interests include surrogate-based modeling and optimization, space mapping, circuit theory, analog signal processing, evolutionary computation, and numerical analysis.

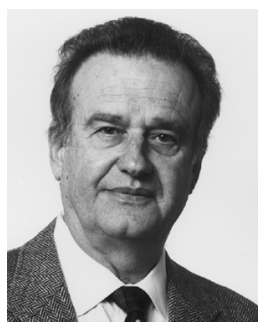

John W. Bandler (S'66-M'66-SM'74-F'78LF'06) studied at Imperial College. He received the B.Sc. (Eng.), Ph.D., and D.Sc. (Eng.) degrees from the University of London, London, U.K., in 1963, 1967, and 1976, respectively.

In 1969, he joined McMaster University, Hamilton, ON, Canada. He is currently a Professor Emeritus. He was President of Optimization Systems Associates Inc., which he founded in 1983, until November 20, 1997, the date of acquisition by the Hewlett-Packard Company. He is President of Bandler Corporation, Dundas, ON, Canada, which he founded in 1997.

Dr. Bandler is a Fellow of several societies including the Royal Society of Canada. He was the recipient of the 2004 IEEE Microwave Theory and Techniques Society (IEEE MTT-S) Microwave Ap The city is 'Hjørring'

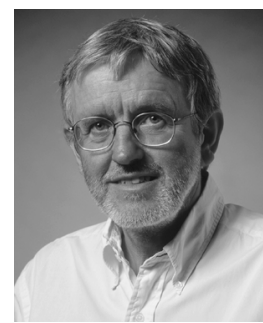

Kaj Madsen was born in [City,] Denmark, in 1943. He undertook his basic education in mathematics at the University of Aarhus, Aarhus, Denmark. He received the Dr.Techn. degree from the Technical University of Denmark (DTU), Lyngby, Copenhagaen, Denmark, in [Year].

He has spent his career with DT/ and Copenhagen University. He is currently a Aull Professor with DTU. He was a main force The year is 1986

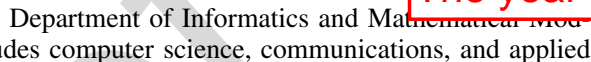
elling, DTU, which includes computer science, communications, and applied mathematics, and since 1995, has headed the department. His primary research interests are nonlinear optimization including space-mapping techniques and global optimization using interval analysis. 


\title{
Space Mapping With Adaptive Response Correction for Microwave Design Optimization
}

\author{
Slawomir Koziel, Senior Member, IEEE, John W. Bandler, Life Fellow, IEEE, and Kaj Madsen
}

\begin{abstract}
Output space mapping is a technique introduced to enhance the robustness of the space-mapping optimization process in case the space-mapped coarse model cannot provide sufficient matching with the fine model. The technique often works very well; however, in some cases it fails. Especially in the microwave area where the typical model response (e.g., $\left|S_{21}\right|$ ) is a highly nonlinear function of the free parameter (e.g., frequency), the output spacemapping correction term may actually increase the mismatch between the surrogate and fine models for points other than the one at which the term was calculated, as in the surrogate model optimization process. In this paper, an adaptive response correction scheme is presented to work in conjunction with space-mapping optimization algorithms. This technique is designed to alleviate the difficulties of the standard output space mapping by adaptive adjustment of the response correction term according to the changes of the space-mapped coarse model response. Examples indicate the robustness of our approach.
\end{abstract}

Index Terms-Engineering optimization, microwave design, response correction, space mapping, space-mapping optimization.

\section{INTRODUCTION}

$\mathbf{S}$ PACE mapping has been used for solving difficult optimization problems in the microwave area for more than a decade [1]-[4]. This methodology is founded on the idea of the optimization of expensive or "fine" models by means of the iterative optimization and updating of so-called "coarse" models which are less accurate, but cheaper to evaluate. Provided that the misalignment between the fine and coarse model is not significant, space-mapping-based algorithms typically provide excellent results after only a few evaluations of the fine model. A similar idea is shared by other surrogate-model-based methods [5]-[11]; however, many of them do not use a simplified physically based coarse model: a functional surrogate is created by direct approximation of the available fine model data.

Space mapping is widely used in the optimization of microwave devices [1]-[3], [12]-[16], where fine models are

Manuscript received October 31, 2007; revised May 25, 2008. This work was supported in part by the Natural Sciences and Engineering Research Council of Canada (NSERC) under Grant RGPIN7239-06 and Grant STPGP336760-06, and by Bandler Corporation.

S. Koziel is with the School of Science and Engineering, Reykjavík University, Reykjavík IS-103, Iceland (e-mail: koziel@ru.is).

J. W. Bandler is with the Simulation Optimization Systems Research Laboratory, Department of Electrical and Computer Engineering, McMaster University, Hamilton, ON, Canada L8S 4K1, and also with Bandler Corporation, Dundas, ON, Canada L9H 5E7 (e-mail: bandler@mcmaster.ca).

$\mathrm{K}$. Madsen is with Informatics and Mathematical Modelling, Technical University of Denmark, DK-2800 Lyngby, Denmark (e-mail: km@imm.dtu.dk).

Color versions of one or more of the figures in this paper are available online at http://ieeexplore.ieee.org.

Digital Object Identifier 10.1109/TMTT.2008.2011243 often based on full-wave electromagnetic simulations, whereas coarse models may be physically based circuit models. Recently, space-mapping techniques have been applied to design problems in a growing number of areas (e.g., [17]-[19]).

A number of papers cover different aspects of space mapping, including the development of new algorithms [2], [3], [20], [21], space-mapping-based modeling [22]-[24], neuro-spacemapping [25]-[28], theoretical foundations [20], [29], [30], etc.

One of the recent developments in space mapping is the so-called output space mapping [29], [31] in which the space-mapping surrogate model is created by enhancing the coarse model using a correction term, typically additive or multiplicative, that allows us to obtain a perfect matching between the fine model and a surrogate at the current iteration point. If sensitivity information is employed, the correction term can be constructed in such a way that the surrogate model matches the fine model with respect to response and first-order derivatives. If equipped with a trust region method [32], the output space-mapping algorithm with the surrogate satisfying first-order consistency conditions can be shown to converge to a local optimum of the fine model [20], [33]. Different versions of output space mapping have been described in the literature, e.g., [4], [20], [29].

Typically, output space mapping improves the performance of the space-mapping algorithm when applied as an auxiliary mapping. If the range of the coarse model is substantially different from the range of the fine model, output space mapping is practically mandatory to make the space-mapping algorithm perform reasonably well.

Unfortunately, in some cases output space mapping does not work as expected. Especially in the microwave area, the typical model response, e.g., the $\left|S_{21}\right|$ parameter, is a highly nonlinear function of the free parameter (typically the frequency of the input signal). In particular, the response may contain sharp minima corresponding to zeros of the transfer function of the underlying device. In such cases, the output space-mapping correction term may actually increase the apparent mismatch between the surrogate and fine model for points other than the one at which the term was calculated, i.e., during the process of surrogate model optimization. It may also severely deform the surrogate model response (see Section II for examples). As a consequence, the performance of the space-mapping algorithm may be degraded both with respect to the quality of the final solution as well as the computational cost of the optimization process.

In this paper, we propose an adaptive response correction scheme, which is designed to alleviate the difficulties of the standard output space mapping and improve the overall performance of the output space mapping. Our technique is based 
on the adaptive adjustment of the response correction term. The adjustment is related to the changes of the space-mapped coarse model response, which occurs during the optimization of the surrogate. More specifically, the model response will be normally shifted, "squeezed" and/or "expanded" in frequency during the optimization process where we adjust the design parameters in order to have the model meet the design specifications. The output space-mapping correction term is kept constant (i.e., independent of the design variables) during the surrogate model optimization according to the standard approach. According to the adaptive response correction approach the correction term is being modified to make it change in frequency in the same way as the space-mapped coarse model response is changing. This technique is based on the assumption that the frequency responses of both the fine and space-mapped coarse model responses are modified in a similar way when the respective models are subjected to the same modification of the design parameters.

The robustness of the adaptive response correction method is demonstrated using microwave design optimization problems.

\section{AdAPTIVE RESPONSE CORRECTION}

\section{A. Motivation}

Let $\boldsymbol{R}_{f}: X_{f} \rightarrow R^{m}, X_{f} \subseteq R^{n}$, and $\boldsymbol{R}_{c}: X_{c} \rightarrow R^{m}, X_{c} \subseteq$ $R^{n}$ denote the response vectors of the fine and coarse models of a given microwave device, respectively. $R$ denotes the set of real numbers. Components of $\boldsymbol{R}_{f}$ and $\boldsymbol{R}_{c}$ may be evaluations of $\left|S_{21}\right|$ parameter at $m$ different frequencies. In particular, we may have $\boldsymbol{R}_{f}(\boldsymbol{x})=\left[R_{f}\left(\boldsymbol{x}, \omega_{1}\right) R_{f}\left(\boldsymbol{x}, \omega_{2}\right) \ldots R_{f}\left(\boldsymbol{x}, \omega_{m}\right)\right]^{T}$, where $R_{f}\left(\boldsymbol{x}, \omega_{j}\right)$ is a component of $\boldsymbol{R}_{f}$ corresponding to frequency $\omega_{j} ; \boldsymbol{x}$ denotes the vector of design parameters of the device.

The space-mapping optimization algorithm produces a sequence of points $\boldsymbol{x}^{(i)}, i=0,1,2, \ldots$, where $\boldsymbol{x}^{(i)}$ represents values of the design parameters (also called design variable vector) at iteration $i ; \boldsymbol{x}^{(i+1)}$ is an optimal solution (design) of the so-called surrogate model $\boldsymbol{R}_{s}^{(i)}$. Typically, $\boldsymbol{x}^{(0)}$ is the optimal solution of the coarse model. Surrogate model at iteration $i, \boldsymbol{R}_{s}^{(i)}$ is based on the coarse model and certain auxiliary mappings. Typically, output space mapping is used on the top of other space-mapping types. We shall use symbol $\overline{\boldsymbol{R}}_{c}^{(i)}$ to denote the space-mapped coarse model at iteration $i$, i.e., the coarse model composed with space mapping (or composition of more mappings if necessary; however, excluding the output space mapping that will be considered separately). For the sake of example, consider input space mapping, in which case $\overline{\boldsymbol{R}}_{c}^{(i)}(\boldsymbol{x})=\boldsymbol{R}_{c}\left(\boldsymbol{B}^{(i)} \cdot \boldsymbol{x}+\boldsymbol{c}^{(i)}\right)$, where $\boldsymbol{B}^{(i)}$ and $\boldsymbol{c}^{(i)}$ are mapping parameters obtained though parameter extraction [20]; $\boldsymbol{B}^{(i)}$ is an $n \times n$ matrix, while $\boldsymbol{c}^{(i)}$ is an $n \times 1$ vector.

The standard output space-mapping technique [29] assumes that the surrogate model $\boldsymbol{R}_{s}^{(i)}$ is defined as

$$
\boldsymbol{R}_{s}^{(i)}(\boldsymbol{x})=\overline{\boldsymbol{R}}_{c}^{(i)}(\boldsymbol{x})+\boldsymbol{d}^{(i)}
$$

with

$$
\boldsymbol{d}^{(i)}=\boldsymbol{R}_{f}\left(\boldsymbol{x}^{(i)}\right)-\overline{\boldsymbol{R}}_{c}^{(i)}\left(\boldsymbol{x}^{(i)}\right)
$$

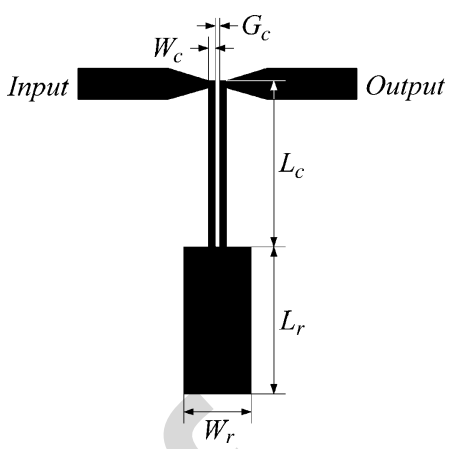

Fig. 1. Geometry of the wideband bandstop microstrip filter [34].

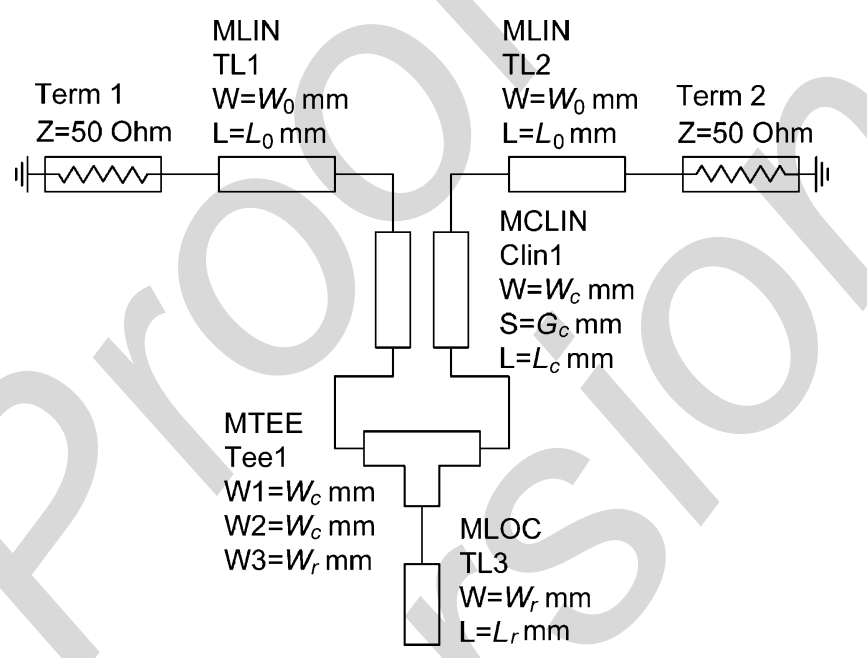

Fig. 2. Coarse model of the wideband bandstop filter (Agilent Technologies' ADS).

This definition ensures a zero-order consistency condition between the fine model and the space-mapping surrogate at $\boldsymbol{x}^{(i)}$, i.e., $\boldsymbol{R}_{f}\left(\boldsymbol{x}^{(i)}\right)=\boldsymbol{R}_{s}^{(i)}\left(\boldsymbol{x}^{(i)}\right)$.

Consider the following example: the wideband bandstop microstrip filter [34] shown in Fig. 1. The design parameters are $\boldsymbol{x}=\left[L_{r} W_{r} L_{c} W_{c} G_{c}\right]^{T}$. The fine model $\boldsymbol{R}_{f}$ is simulated in FEKO [35]. The coarse model $\boldsymbol{R}_{c}$ is the circuit model implemented in Agilent Technologies' Advanced Design System (ADS) [36] (Fig. 2).

The design specifications are

$$
\begin{aligned}
& \left|S_{21}\right| \geq-3 \mathrm{~dB}, \quad \text { for } 1.0 \mathrm{GHz} \leq \omega \leq 2.0 \mathrm{GHz} \\
& \left|S_{21}\right| \leq-20 \mathrm{~dB}, \quad \text { for } 3.0 \mathrm{GHz} \leq \omega \leq 9.0 \mathrm{GHz} \\
& \left|S_{21}\right| \geq-3 \mathrm{~dB}, \quad \text { for } 10.0 \mathrm{GHz} \leq \omega \leq 11.0 \mathrm{GHz} .
\end{aligned}
$$

For this problem we use the input-space-mapped coarse model defined as $\overline{\boldsymbol{R}}_{c}^{(i)}(\boldsymbol{x})=\boldsymbol{R}_{c}\left(\boldsymbol{x}+\boldsymbol{c}^{(i)}\right)$, where vector $\boldsymbol{c}^{(i)}$ is obtained using parameter extraction. On top of $\overline{\boldsymbol{R}}_{c}^{(i)}$, we use standard output space mapping, as defined in (1), (2).

Fig. 3 shows the fine and space-mapped coarse model responses at the starting point $\boldsymbol{x}^{(0)}=\left[\begin{array}{lllll}7.017 & 0.980 & 8.653 & 0.055 & 0.109\end{array}\right]^{T} \mathrm{~mm}$ (the coarse model optimal solution). Fig. 4 shows the response of the initial surrogate model $\boldsymbol{R}_{s}^{(0)}$ at the starting point and at its optimal solution $\boldsymbol{x}^{(1)}$. We can observe that $\boldsymbol{R}_{s}^{(0)}\left(\boldsymbol{x}^{(1)}\right)$ is substantially distorted around $6 \mathrm{GHz}$, which is a consequence 


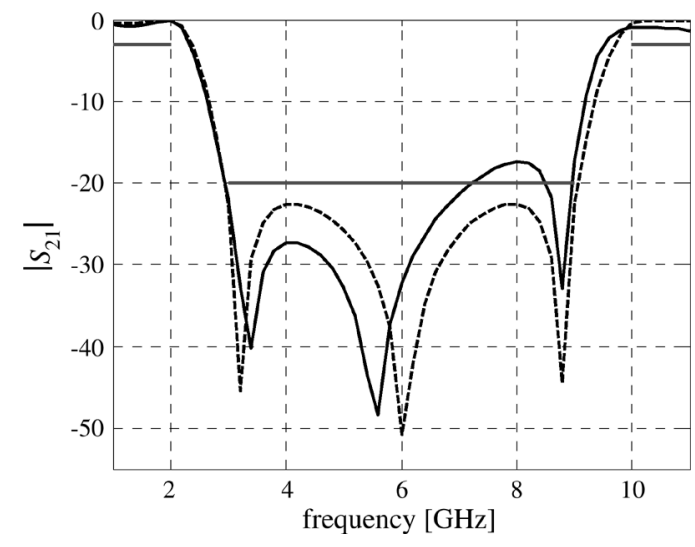

Fig. 3. Wideband bandstop filter: fine model (solid line) and space-mapped coarse model (dashed line) responses at $\boldsymbol{x}^{(0)}$.

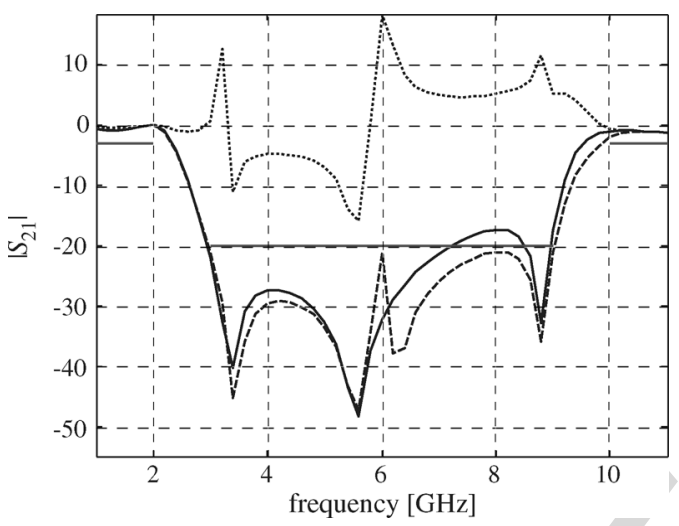

Fig. 4. Wideband bandstop filter: initial surrogate model response $\boldsymbol{R}_{\mathrm{s}}^{(0)}\left(\boldsymbol{x}^{(0)}\right)$ (solid line), optimized surrogate model response $\boldsymbol{R}_{s}^{(0)}\left(\boldsymbol{x}^{(1)}\right)$ (dashed line), and standard space-mapping correction term $\boldsymbol{d}^{(0)}$ (dotted line).

of the fact that the output space-mapping correction term $\boldsymbol{d}^{(0)}$ (shown in Fig. 4 as a dotted line) is a vector (independent of the design variables $\boldsymbol{x}$ ) with a sharp maximum around $6 \mathrm{GHz}$, while it is seen that the surrogate model optimization process tends to move the central zero of the filter into higher frequencies. In other words, the distortion due to the correction term $\boldsymbol{d}^{(0)}$ is a source of difficulty in the surrogate model optimization process.

The fine model specification error at $\boldsymbol{x}^{(0)}$ is $+2.7 \mathrm{~dB}$. Due to the above-mentioned problems, space-mapping optimization finds a solution with corresponding specification error $-1.1 \mathrm{~dB}$, which is rather poor as the minimax optimum for this problem is about $-2 \mathrm{~dB}$. The number of fine model evaluations required to find the solution (excluding evaluation at $\boldsymbol{x}^{(0)}$ ) is 8 .

\section{B. Adaptive Response Correction: Concept}

In order to avoid the problems highlighted above, we propose the following adaptive response correction technique. The surrogate model $\boldsymbol{R}_{s}^{(i)}$ at any iteration point $\boldsymbol{x}^{(i)}$ is defined as

$$
\boldsymbol{R}_{s}^{(i)}(\boldsymbol{x})=\overline{\boldsymbol{R}}_{c}^{(i)}(\boldsymbol{x})+\Delta_{r}\left(\boldsymbol{x}, \boldsymbol{x}^{(i)}\right)
$$

where $\boldsymbol{\Delta}_{r}\left(\boldsymbol{x}, \boldsymbol{x}^{(i)}\right)$ is the response correction term dependent on the design variables $x$. As we still want to maintain a perfect

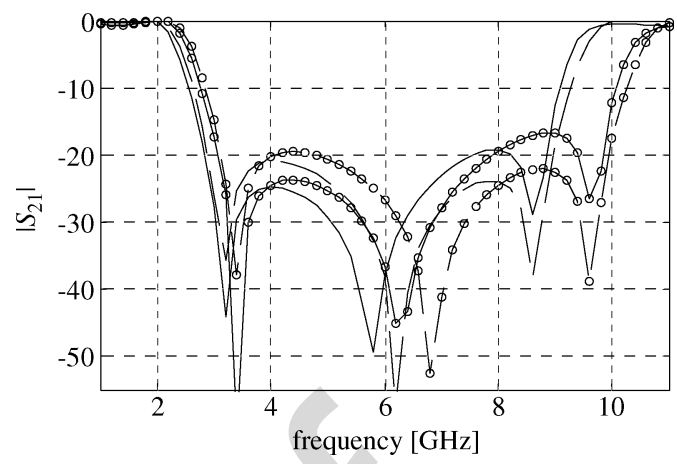

Fig. 5. Wideband bandstop filter: fine (solid line) and space-mapped coarse model (dashed line) responses at certain point (design variable vector), as well as fine (solid line with circle markers) and space-mapped coarse model (dashed line with circle markers) responses at a different point.

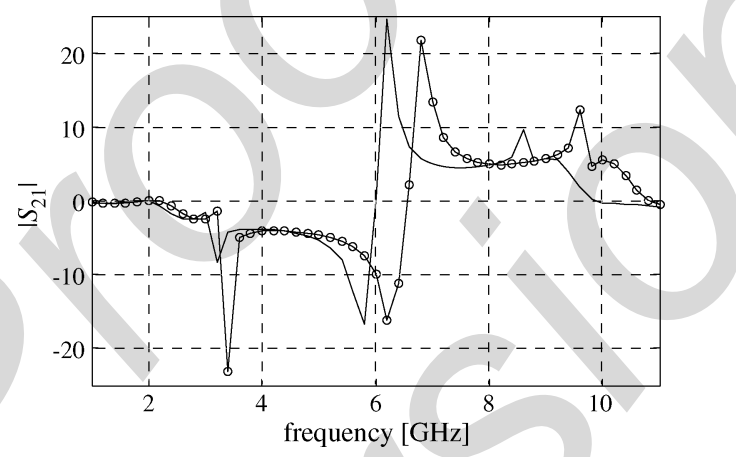

Fig. 6. Wideband bandstop filter: standard output space-mapping correction terms corresponding to responses shown in Fig. 5.

match between the fine model and the space-mapping surrogate at $\boldsymbol{x}^{(i)}, \boldsymbol{\Delta}_{r}\left(\boldsymbol{x}, \boldsymbol{x}^{(i)}\right)$ must satisfy

$$
\boldsymbol{\Delta}_{r}\left(\boldsymbol{x}^{(i)}, \boldsymbol{x}^{(i)}\right)=\boldsymbol{d}^{(i)}=\boldsymbol{R}_{f}\left(\boldsymbol{x}^{(i)}\right)-\overline{\boldsymbol{R}}_{c}^{(i)}\left(\boldsymbol{x}^{(i)}\right) .
$$

The idea behind the adaptive response correction is to account for the difference between the space-mapped coarse model response at $\boldsymbol{x}$ and at $\boldsymbol{x}^{(i)}$ and to modify the correction term accordingly with the initial correction $\boldsymbol{d}^{(i)}$ taken as a reference. In particular, we want to modify the initial correction term $\boldsymbol{d}^{(i)}$ in such a way that this modification reflects changes to $\overline{\boldsymbol{R}}_{c}^{(i)}$ during the process of surrogate model optimization. In particular, if the response of $\overline{\boldsymbol{R}}_{c}^{(i)}$ shifts or changes its shape with respect to frequency, the response correction term should track these changes.

As an illustration, consider Fig. 5, which shows the fine and space-mapped coarse model responses for the wideband bandstop filter (Section II-A) at two different points. It is seen that the general relation between the mapped coarse model response and its corresponding fine model response is preserved (e.g., the center minimum of the mapped coarse model response is at higher frequency than the corresponding minimum for the fine model). Fig. 6 shows the standard output space-mapping correction terms $\boldsymbol{d}$ corresponding to the responses shown in Fig. 5. As we can observe, the relation between these terms as functions of frequency is similar to the relation between the space-mapped coarse model responses so that proper tracking of coarse model changes help determine necessary changes to the output space-mapping correction terms. 
In our implementation, the tracking of the coarse model response changes is realized through a proper mapping that describes the shape relations between two coarse model responses and updates the response correction term so that the initial and current correction terms correspond to the same (frequencybased) relationship as the initial and current coarse model responses. A rigorous description and technical details of this process are given below.

Let us assume without loss of generality that the components of $\boldsymbol{R}_{f}\left(\boldsymbol{R}_{c}\right)$ correspond to the model evaluations (e.g., $\left|S_{21}\right|$ ) at $m$ different frequency points, i.e., $\Omega=\left[\omega_{1} \omega_{2} \ldots \omega_{m}\right]^{T}$. For example, we have $\boldsymbol{R}_{f}(\boldsymbol{x})=$ $\left[R_{f}\left(\boldsymbol{x}, \omega_{1}\right) R_{f}\left(\boldsymbol{x}, \omega_{2}\right) \ldots R_{f}\left(\boldsymbol{x}, \omega_{m}\right)\right]^{T}$, where $R_{f}\left(\boldsymbol{x}, \omega_{j}\right)$ is a component of $\boldsymbol{R}_{f}$ corresponding to frequency $\omega_{j}$. Let $I: R \times \Omega \times\left[\omega_{\min }, \omega_{\max }\right] \rightarrow R$ be an interpolation/extrapolation function such that $I(R, \Omega, \cdot)$ interpolates the response $R$ defined on $\Omega$ onto $\left[\omega_{1}, \omega_{m}\right]$ and extrapolates $R$ onto $\left[\omega_{\min }, \omega_{1}\right)$ and $\left(\omega_{m}, \omega_{\text {max }}\right]$. In particular, $I(\boldsymbol{R}, \Omega, \omega)$ is the evaluation of the interpolated/extrapolated response $R$ at frequency $\omega$. In this paper, we use $I$ implemented as piecewise cubic splines (interpolation part) and linear extrapolation (extrapolation part).

The core of the adaptive response correction is a mapping $F^{(i)}: X_{q} \times\left[\omega_{1}, \omega_{m}\right] \rightarrow\left[\omega_{\min }, \omega_{\max }\right] . F^{(i)}$ is established at iteration $i$ in such a way that the difference between $\overline{\boldsymbol{R}}_{c}^{(i)}(\boldsymbol{x})$, i.e., the space-mapped coarse model response at $\boldsymbol{x}$ and $I\left(\overline{\boldsymbol{R}}_{c}^{(i)}\left(\boldsymbol{x}^{(i)}\right), \Omega, F^{(i)}(\boldsymbol{x}, \Omega)\right)$, i.e., $\overline{\boldsymbol{R}}_{c}^{(i)}\left(\boldsymbol{x}^{(i)}\right)$ interpolated/extrapolated through $I$ onto $\left[\omega_{\min }, \omega_{\max }\right]$ and evaluated at frequencies $F^{(i)}\left(\boldsymbol{x}, \omega^{(1)}, F^{(i)}\left(\boldsymbol{x}, \omega_{2}\right), \ldots, F^{(i)}\left(\boldsymbol{x}, \omega_{m}\right)\right.$ is minimized. Here, $X_{q}$ denotes the space-mapped coarse model domain. $F^{(i)}(\boldsymbol{x}, \Omega)$ will be referred to as mapped frequency.

In general, $\omega_{\min }\left(\omega_{\max }\right)$ may differ from $\omega_{1}\left(\omega_{m}\right)$, respectively. If the response of the model is not a constant function of frequency around $\omega_{1}\left(\omega_{m}\right)$, one may need to allow the mapped frequencies exceed the interval $\left[\omega_{1}, \omega_{m}\right]$ in order to make the good alignment between $\overline{\boldsymbol{R}}_{c}^{(i)}(\boldsymbol{x})$ and $I\left(\overline{\boldsymbol{R}}_{c}^{(i)}\left(\boldsymbol{x}^{(i)}\right), \Omega, F^{(i)}(\boldsymbol{x}, \Omega)\right)$ possible. For example, if $\overline{\boldsymbol{R}}_{c}^{(i)}(\boldsymbol{x})$ is larger than $\overline{\boldsymbol{R}}_{c}^{(i)}\left(\boldsymbol{x}^{(i)}\right)$ at $\omega_{1}$ and both are decreasing functions of frequency around $\omega_{1}$, the only way to make it possible to align $\overline{\boldsymbol{R}}_{c}^{(i)}(\boldsymbol{x})$ with $I\left(\overline{\boldsymbol{R}}_{c}^{(i)}\left(\boldsymbol{x}^{(i)}\right), \Omega, F^{(i)}(\boldsymbol{x}, \Omega)\right)$ at $\omega_{1}$ would be to allow $\omega_{\min }<\omega_{1}$ so that $F^{(i)}\left(\boldsymbol{x}, \omega_{1}\right)<\omega_{1}$ and $I\left(\overline{\boldsymbol{R}}_{c}^{(i)}\left(\boldsymbol{x}^{(i)}\right), \Omega, F^{(i)}(\boldsymbol{x}, \Omega)\right)$ at $\omega_{1}$ would refer to $\overline{\boldsymbol{R}}_{c}^{(i)}\left(\boldsymbol{x}^{(i)}\right)$ for frequencies smaller than $\omega_{1}$. In practice, we may want to set $\omega_{\min }$ to be slightly smaller than $\omega_{1}$ ( $\omega_{\max }$ to be slightly larger than $\left.\omega_{m}\right)$.

The vector $I\left(\overline{\boldsymbol{R}}_{c}^{(i)}\left(\boldsymbol{x}^{(i)}\right), \Omega, F^{(i)}(\boldsymbol{x}, \Omega)\right)$ is nothing but $\overline{\boldsymbol{R}}_{c}^{(i)}\left(\boldsymbol{x}^{(i)}\right)$ scaled with respect to frequency in order to be as similar to $\overline{\boldsymbol{R}}_{c}^{(i)}(\boldsymbol{x})$ as possible in a given sense. In other words, mapping $F^{(i)}$ is supposed to be defined in such a way that mapped frequency $F^{(i)}(x, \Omega)$ reflects the shape change of the space-mapped coarse model response at $\boldsymbol{x}$ with respect to its original shape at $\boldsymbol{x}^{(i)}$. For the sake of consistency with condition (4), $F^{(i)}\left(x^{(i)}, \Omega\right)$ should be equal $\Omega$, i.e., the mapped frequency should be the same as the original frequency at $\boldsymbol{x}^{(i)}$.
Having the mappings $I$ and $F^{(i)}$, we can define the response correction term as follows:

$\boldsymbol{\Delta}_{r}\left(\boldsymbol{x}, \boldsymbol{x}^{(i)}\right)=I\left(\boldsymbol{R}_{f}\left(\boldsymbol{x}^{(i)}\right)-\overline{\boldsymbol{R}}_{c}^{(i)}\left(\boldsymbol{x}^{(i)}\right), \Omega, F^{(i)}(\boldsymbol{x}, \Omega)\right)$

The reason for using function $I$ is the fact that the entire process requires information about $\overline{\boldsymbol{R}}_{c}^{(i)}\left(\boldsymbol{x}^{(i)}\right)$ and $\boldsymbol{R}_{f}\left(\boldsymbol{x}^{(i)}\right)-$ $\overline{\boldsymbol{R}}_{c}^{(i)}\left(\boldsymbol{x}^{(i)}\right)$ at frequencies different from the original evaluation frequencies $\omega_{1}, \omega_{2}, \ldots, \omega_{m}$.

In the following sections, we describe two realizations of the mapping $F^{(i)}$. Other possible realizations will be dealt with elsewhere.

\section{Mapping F Implementation: Realization 1}

This realization is suitable for problems in which the coarse model response is rather smooth as a function of frequency (i.e., without sharp local minima and maxima) and such that the range of the response (as a function of frequency) does not change much with $\boldsymbol{x}$.

The mapped frequency $F^{(i)}(x, \omega)$ is defined as

$$
F^{(i)}(x, \omega)=\left[\lambda^{(i)}(x)\right]^{T} \boldsymbol{v}(\omega)
$$

where $\lambda^{(i)}(\boldsymbol{x})=\left[\lambda_{1}^{(i)}(\boldsymbol{x}) \lambda_{2}^{(i)}(\boldsymbol{x}) \ldots \lambda_{p}^{(i)}(\boldsymbol{x})\right]^{T}$ are scaling coefficients determined as

$$
\boldsymbol{\lambda}^{(i)}(\boldsymbol{x})=\arg \min _{\boldsymbol{\lambda}}\left\|I\left(\overline{\boldsymbol{R}}_{c}^{(i)}\left(\boldsymbol{x}^{(i)}\right), \Omega, \boldsymbol{\lambda}^{T} \boldsymbol{v}(\Omega)\right)-\overline{\boldsymbol{R}}_{c}^{(i)}(\boldsymbol{x})\right\|
$$

while $\boldsymbol{v}(\omega)=\left[v_{1}(\omega) v_{2}(\omega) \ldots v_{p}(\omega)\right]^{T}$ are basis functions. Optimization problem (6), which is readily solved by MATLAB, has the following constraints: a) $d\left(\left[\boldsymbol{\lambda}^{(i)}(\boldsymbol{x})\right]^{T} \boldsymbol{v}(\omega)\right) / d \omega>0$, i.e., scaling function $F$ is monotonic; b) $\left[\lambda^{(i)}(\boldsymbol{x})\right]^{T} \boldsymbol{v}\left(\omega_{1}\right) \geq \omega_{\min }$; and c) $\left[\lambda^{(i)}(\boldsymbol{x})\right]^{T} \boldsymbol{v}\left(\omega_{m}\right) \leq \omega_{\max }$, i.e., we need to ensure that $F^{(i)}(\boldsymbol{x}, \omega)$ is a monotonic function of $\omega$ within its range in $\left[\omega_{\min }, \omega_{\max }\right]$. In this paper, we use a third-order polynomial scaling, i.e., we have $p=4$ and $\boldsymbol{v}(\omega)=\left[1 \omega \omega^{2} \omega^{3}\right]^{T}$. Typically $\omega_{\min }$ is slightly smaller than $\omega_{1}$ (e.g., by $10 \%-20 \%$ ), and $\omega_{\max }$ is slightly larger than $\omega_{m}$.

\section{Mapping F Implementation: Realization 2}

This realization is suitable for problems in which the coarse model response contains clearly defined characteristic frequencies, e.g., sharp minima corresponding to zeros of the transfer function.

Let $\Omega_{1}=\left\{\omega_{1.1}, \omega_{1.2}, \ldots, \omega_{1 . N}\right\}$ and $\Omega_{2}=$ $\left\{\omega_{2.1}, \omega_{2.2}, \ldots, \omega_{2 . N}\right\}$ be the sets of characteristic frequencies of $\overline{\boldsymbol{R}}_{c}^{(i)}\left(\boldsymbol{x}^{(i)}\right)$ and $\overline{\boldsymbol{R}}_{c}^{(i)}(\boldsymbol{x})$, respectively. The mapped frequency $F^{(i)}(\boldsymbol{x}, \omega)$ is defined as an interpolation function such that $F^{(i)}\left(\boldsymbol{x}, \omega_{2 . j}\right)=\omega_{1 . j}, j=1,2, \ldots, N$. In this paper, we implement $F$ using cubic splines. If there is a different number of characteristic frequencies for $\overline{\boldsymbol{R}}_{c}^{(i)}(\boldsymbol{x})$ and $\overline{\boldsymbol{R}}_{c}^{(i)}\left(\boldsymbol{x}^{(i)}\right)$, both $\Omega_{1}$ and $\Omega_{2}$ are adjusted using an auxiliary algorithm in order to maintain the correspondence between the two sets. In order to have the mapped frequency $F^{(i)}(\boldsymbol{x}, \omega)$ well defined on $\left[\omega_{1}, \omega_{m}\right]$, we typically set $\omega_{1.1}=\omega_{2.1}=\omega_{1}$ and $\omega_{1 . N}=\omega_{2 . N}=\omega_{m}$. However, if there is a difference between 
TABLE I

SPACE-MAPPING OPTIMIZATION RESUlTS FOR THE WIDEBAND BANDSTOP MICROSTRIP FILTER

\begin{tabular}{ccc}
\hline \hline Output Space Mapping Used & $\begin{array}{c}\text { Final Spec. Error } \\
{[\mathrm{dB}]}\end{array}$ & $\begin{array}{c}\text { Number of Fine Model } \\
\text { Evaluations }\end{array}$ \\
\hline Standard Output Space Mapping & -1.1 & 8 \\
Adaptive Response Correction & -2.1 & 3 \\
\hline \hline
\end{tabular}

* Excludes the fine model evaluation at the starting point.

$\overline{\boldsymbol{R}}_{c}^{(i)}(\boldsymbol{x})$ and $\overline{\boldsymbol{R}}_{c}^{(i)}\left(\boldsymbol{x}^{(i)}\right)$ at $\omega_{1}$, then it is better to set $\omega_{1.1}$ to $\bar{\omega}=\arg \min _{\omega}\left|I\left(\overline{\boldsymbol{R}}_{c}^{(i)}\left(\boldsymbol{x}^{(i)}\right), \Omega, \omega\right)-\bar{R}_{c}^{(i)}\left(\boldsymbol{x}, \omega_{1}\right)\right|$ in order to obtain a better overall match between $I\left(\overline{\boldsymbol{R}}_{c}^{(i)}\left(\boldsymbol{x}^{(i)}\right), \Omega, F^{(i)}(\boldsymbol{x}, \Omega)\right)$ and $\overline{\boldsymbol{R}}_{c}^{(i)}(\boldsymbol{x})$. A similar adjustment can be done for $\omega_{1 . N}$.

In addition to frequency scaling, there is the possibility of scaling the response correction term with respect to its amplitude, also based on the response change of the space-mapped coarse model. In this paper, however, we do not use this approach.

It should be noted that, although we assumed that the model response components are related through a free parameter, such as frequency, the adaptive response correction technique should work even if such a relation is not explicitly stated. One can consider the response component indices as values of the "free" parameter.

\section{EXAMPLES}

\section{A. Wideband Bandstop Filter}

As a first example, we again consider the wideband bandstop filter described in Section II. We optimized this filter using the input-space-mapped coarse model $\overline{\boldsymbol{R}}_{c}^{(i)}(\boldsymbol{x})=\boldsymbol{R}_{c}\left(\boldsymbol{x}+\boldsymbol{c}^{(i)}\right)$ and the adaptive response correction technique of Section II. We used Realization 2 of the scaling function $F$ as the filter response contains three clearly defined characteristic points. Table I shows the optimization results, as well as a comparison with the standard output space mapping. Note that the quality of solution found with the adaptive response correction is better than the one obtained with the standard method. The number of fine model evaluations required to find the solution is also substantially smaller for the adaptive correction technique.

In order to understand the performance of the adaptive response correction technique, consider Fig. 7 showing the response of initial surrogate model $\boldsymbol{R}_{s}^{(0)}$ at the starting point and at its optimal solution $\boldsymbol{x}^{(1)}$. We can observe that, in contrast to the standard output space mapping (cf. Fig. 4), $\boldsymbol{R}_{s}^{(0)}\left(\boldsymbol{x}^{(1)}\right)$ is not significantly distorted, which is because the response correction term is modified to reflect the change of $\overline{\boldsymbol{R}}_{c}^{(i)}\left(\boldsymbol{x}^{(1)}\right)$ with respect to $\overline{\boldsymbol{R}}_{c}^{(i)}\left(\boldsymbol{x}^{(0)}\right)$. Fig. 8 shows the correction terms $\Delta_{r}\left(x^{(0)}, \boldsymbol{x}^{(0)}\right)=\boldsymbol{d}^{(0)}$ and $\boldsymbol{\Delta}_{r}\left(\boldsymbol{x}^{(1)}, \boldsymbol{x}^{(0)}\right)$. Fig. 9 shows the plot of the scaling function $F^{(0)}\left(\boldsymbol{x}^{(1)}, \omega\right)$. The fine model response at the design found by the space-mapping algorithm with the adaptive response correction is shown in Fig. 10.

\section{B. Capacitively Coupled Dual-Behavior Resonator Filter}

Our second example is a second-order capacitively coupled dual-behavior resonator microstrip filter [37] shown in Fig. 11.

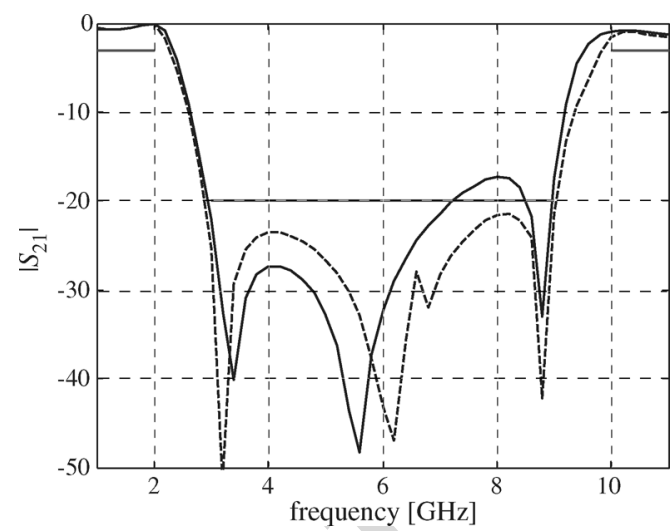

Fig. 7. Wideband bandstop filter: surrogate model response $\boldsymbol{R}_{s}^{(0)}\left(\boldsymbol{x}^{(0)}\right)$ (solid line) and optimized surrogate model response $\boldsymbol{R}_{s}^{(0)}\left(\boldsymbol{x}^{(1)}\right)$ (dashed line). Plots obtained for the adaptive response correction method.

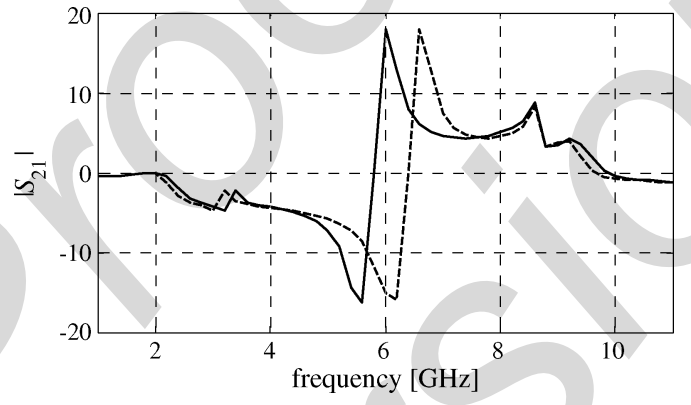

Fig. 8. Wideband bandstop filter: correction terms $\Delta_{r}\left(\boldsymbol{x}^{(0)}, \boldsymbol{x}^{(0)}\right)$ (solid line), and $\Delta_{r}\left(\boldsymbol{x}^{(1)}, \boldsymbol{x}^{(0)}\right)$ (dashed line). Plots obtained for the adaptive response correction method.

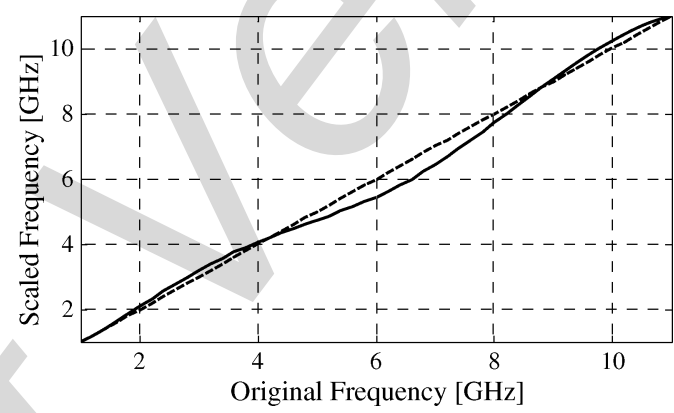

Fig. 9. Wideband bandstop filter: scaling function $F^{(0)}\left(\boldsymbol{x}^{(1)}, \omega\right)$ (solid line). Plot obtained for the adaptive response correction method. The identity function plot is shown as dashed line.

The design parameters are $\boldsymbol{x}=\left[\begin{array}{llll}L_{1} & L_{2} & L_{3} & S\end{array}\right]^{T}$. The fine model $\boldsymbol{R}_{f}$ is simulated in FEKO [35]. The coarse model $\boldsymbol{R}_{c}$ is the circuit model implemented in Agilent Technologies' ADS [36] and shown in Fig. 12. The design specifications for the filter are

$$
\begin{aligned}
& \left|S_{21}\right| \leq-20 \mathrm{~dB}, \quad \text { for } 2.0 \mathrm{GHz} \leq \omega \leq 3.2 \mathrm{GHz} \\
& \left|S_{21}\right| \geq-3 \mathrm{~dB}, \quad \text { for } 3.8 \mathrm{GHz} \leq \omega \leq 4.2 \mathrm{GHz} \\
& \left|S_{21}\right| \leq-20 \mathrm{~dB}, \quad \text { for } 4.8 \mathrm{GHz} \leq \omega \leq 6.0 \mathrm{GHz} .
\end{aligned}
$$

For this problem, we use the input-space-mapped coarse model $\overline{\boldsymbol{R}}_{c}^{(i)}(\boldsymbol{x})=\boldsymbol{R}_{c}\left(\boldsymbol{x}+\boldsymbol{c}^{(i)}\right)$ enhanced by a frequency space mapping in which the coarse model is evaluated at a frequency different than the fine model according to the affine mapping 


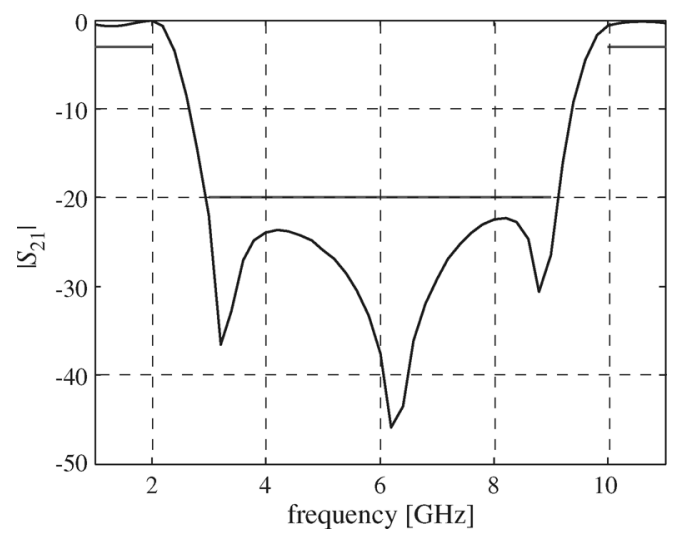

Fig. 10. Wideband bandstop filter: fine model response at the design found by the space-mapping optimization algorithm with the adaptive response correction (Realization 2).

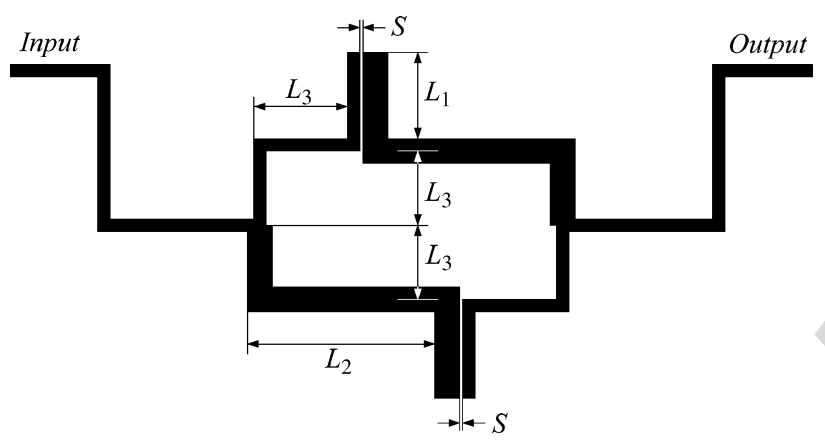

Fig. 11. Geometry of the capacitively coupled dual-behavior resonator microstrip filter [37].

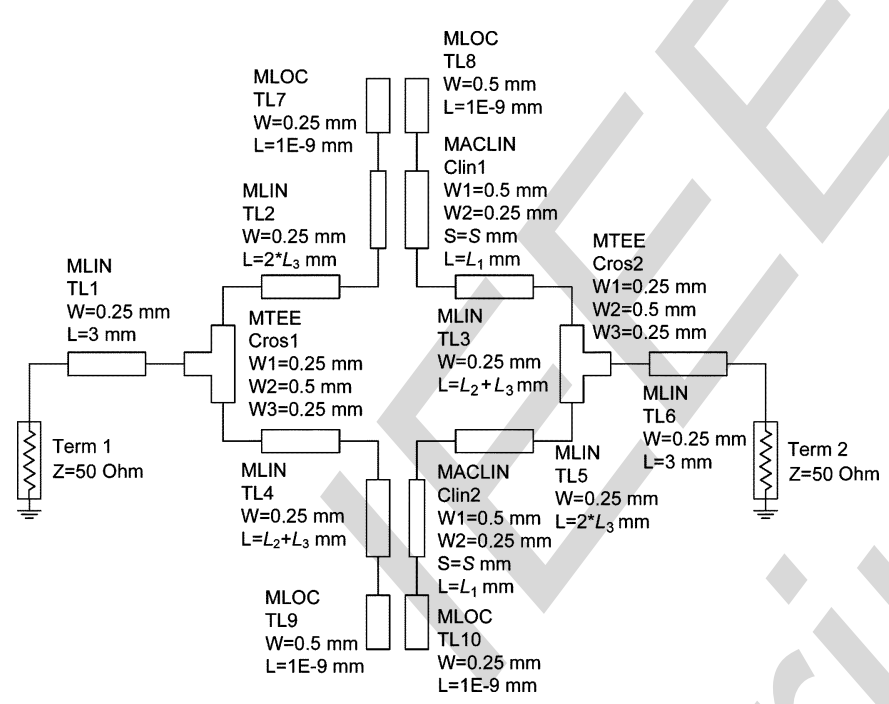

Fig. 12. Coarse model of the capacitively coupled dual-behavior resonator microstrip filter (Agilent Technologies' ADS).

$\omega \leftarrow f_{1}+f_{2} \omega$. Both $\boldsymbol{c}^{(i)}$ and $\boldsymbol{F}^{(i)}=\left[f_{1} f_{2}\right]$ are found at iteration $i$ using parameter extraction. The starting point is the coarse model optimal solution $\boldsymbol{x}^{(0)}=[2.4156 .0931 .1670 .082]^{T} \mathrm{~mm}$. Fig. 13 shows the coarse and fine model responses at $\boldsymbol{x}^{(0)}$. The fine model specification error at $\boldsymbol{x}^{(0)}$ equals $+7.8 \mathrm{~dB}$.

Space-mapping optimization was performed three times using the space-mapped coarse model $\overline{\boldsymbol{R}}_{c}^{(i)}$ with: 1) the standard output space mapping (1), (2); 2) Realization 1 of the

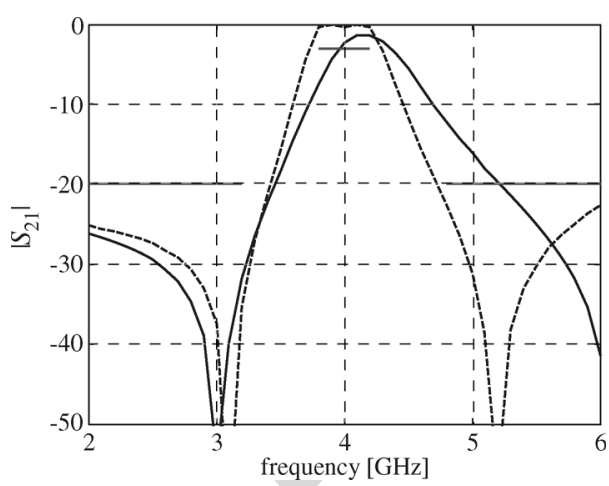

Fig. 13. Capacitively coupled dual-behavior resonator microstrip filter: coarse (dashed line) and fine (solid line) model response at the starting point $\boldsymbol{x}^{(0)}$.

TABLE II

SPACE-MAPPING OPTIMIZATION RESULTS FOR THE CAPACITIVELY COUPLED MICROSTRIP FILTER

\begin{tabular}{ccc}
\hline \hline Output Space Mapping Used & $\begin{array}{c}\text { Final Spec. Error } \\
{[\mathrm{dB}]}\end{array}$ & $\begin{array}{c}\text { Number of Fine Model } \\
\text { Evaluations }\end{array}$ \\
\hline Standard Output Space Mapping & -1.9 & 8 \\
Adaptive Response Correction & -2.5 & 5 \\
(Realization 1) & $(-2.1)$ & $(3)$ \\
Adaptive Response Correction & -2.0 & 3 \\
(Realization 2) & & \\
\hline \hline
\end{tabular}

* Excludes the fine model evaluation at the starting point.

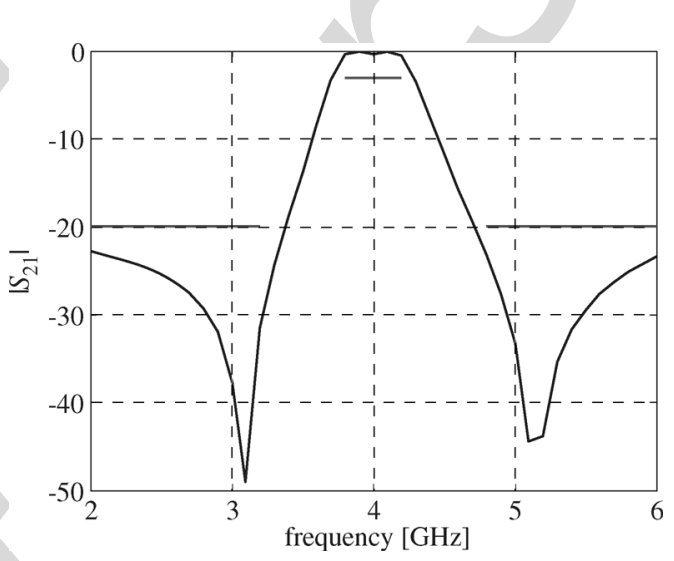

Fig. 14. Capacitively coupled dual-behavior resonator microstrip filter: fine model response at the design found by the space-mapping optimization algorithm with the adaptive response correction (Realization 1).

adaptive response correction technique of Section II; and 3) Realization 2 of the adaptive response correction method.

Table II shows the optimization results for the three methods. The quality of the solution found with the adaptive response correction (both for Realization 1 and Realization 2) is better than the quality of the solution obtained with the standard output space mapping. The number of fine model evaluations required to find the solution is smaller for the adaptive correction technique. It also follows from the results shown in Table II that Realization 1 of the adaptive response correction is slightly more suitable for our filter than Realization 2. Fig. 14 shows the fine model response at the final solution found by the space mapping with Realization 1 of the adaptive response correction. 


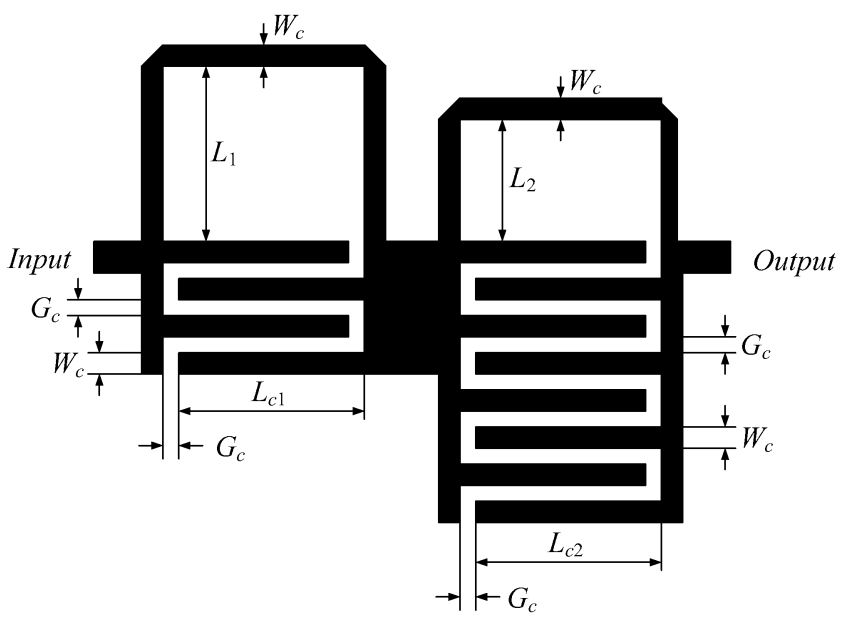

Fig. 15. Geometry of the low-pass elliptic microstrip filter [38].

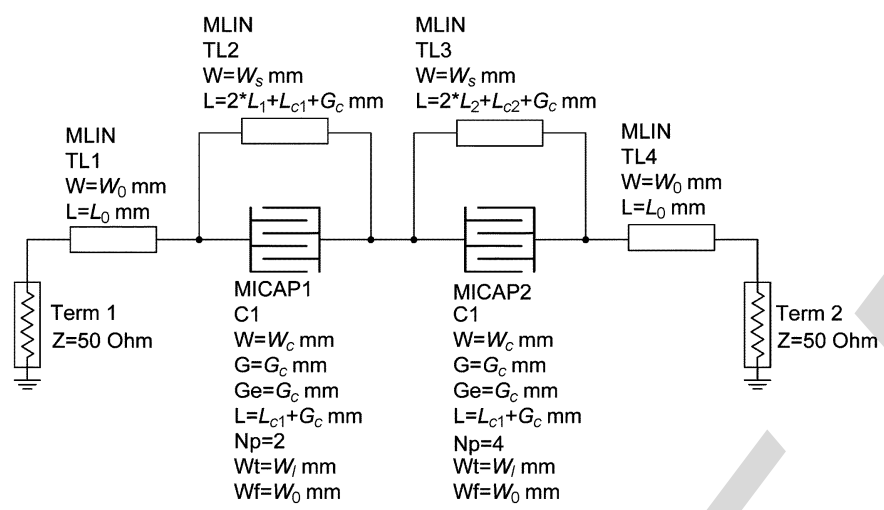

Fig. 16. Coarse model of the low-pass elliptic microstrip filter (Agilent Technologies' ADS).

\section{Two-Section Low-Pass Elliptic Filter}

Our last example is a two-section low-pass elliptic microstrip filter [38] shown in Fig. 15. The design parameters are $\boldsymbol{x}=$ $\left[\begin{array}{llllll}L_{1} & L_{2} & L_{c 1} & L_{c 2} & W_{c} & G_{c}\end{array}\right]^{T}$. The fine model $\boldsymbol{R}_{f}$ is simulated in Sonnet $\boldsymbol{e m}$ [39] using a $0.02 \mathrm{~mm} \times 0.02 \mathrm{~mm}$ grid size. The coarse model $\boldsymbol{R}_{c}$ is the circuit model implemented in Agilent Technologies' ADS [36] and shown in Fig. 16. The design specifications for the elliptic low-pass filter are

$$
\begin{aligned}
& \left|S_{21}\right| \geq-3 \mathrm{~dB}, \quad \text { for } 1.0 \mathrm{GHz} \leq \omega \leq 2.0 \mathrm{GHz} \\
& \left|S_{21}\right| \geq-20 \mathrm{~dB}, \quad \text { for } 2.3 \mathrm{GHz} \leq \omega \leq 4.0 \mathrm{GHz} .
\end{aligned}
$$

For this problem, we use the input-space-mapped coarse model $\overline{\boldsymbol{R}}_{c}^{(i)}(\boldsymbol{x})=\boldsymbol{R}_{c}\left(\boldsymbol{x}+\boldsymbol{c}^{(i)}\right)$, where $\boldsymbol{c}^{(i)}$ is obtained at iteration $i$ using parameter extraction.

The starting point is the coarse model optimal solution $x^{(0)}=$ [2.08 0.52 .763 .220 .10 .1$]^{T} \mathrm{~mm}$. Fig. 17 shows the coarse and fine model responses at $\boldsymbol{x}^{(0)}$. The fine model specification error at $x^{(0)}$ equals $+6.4 \mathrm{~dB}$.

We performed the space-mapping optimization of the low-pass elliptic filter twice, first using the input-space-mapped coarse model together with the standard output space mapping, and then using the mapped coarse model with the adaptive response correction method. We used Realization 2 as the filter response contains two clearly defined characteristic points

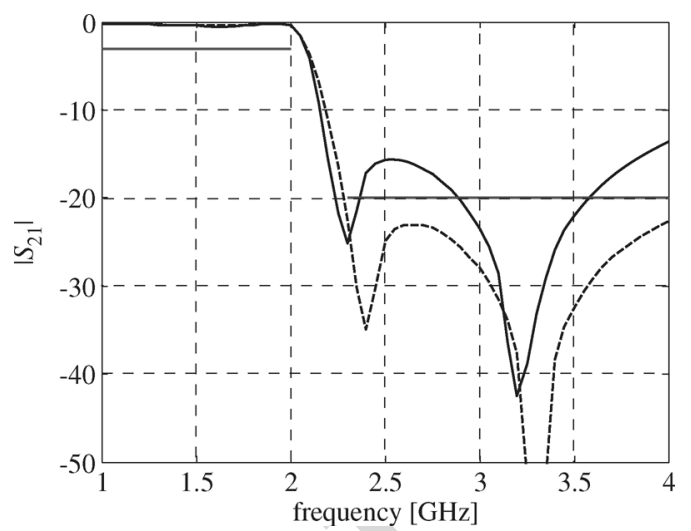

Fig. 17. Low-pass elliptic filter: coarse (dashed line) and fine (solid line) model response at the starting point $\boldsymbol{x}^{(0)}$.

TABLE III

SPACE-MAPPING OPTIMIZATION RESULTS FOR THE LOW-PASS ELLIPTIC MICROSTRIP FILTER

\begin{tabular}{lcc}
\hline \hline Output Space Mapping Used & $\begin{array}{c}\text { Final Spec. Error } \\
{[\mathrm{dB}]}\end{array}$ & $\begin{array}{c}\text { Number of Fine Model } \\
\text { Evaluations* }\end{array}$ \\
\hline $\begin{array}{l}\text { Standard Output Space Mapping } \\
\text { Adaptive Response Correction }\end{array}$ & -1.5 & 8 \\
\hline \hline
\end{tabular}

$*$ Excludes the fine model evaluation at the starting point.

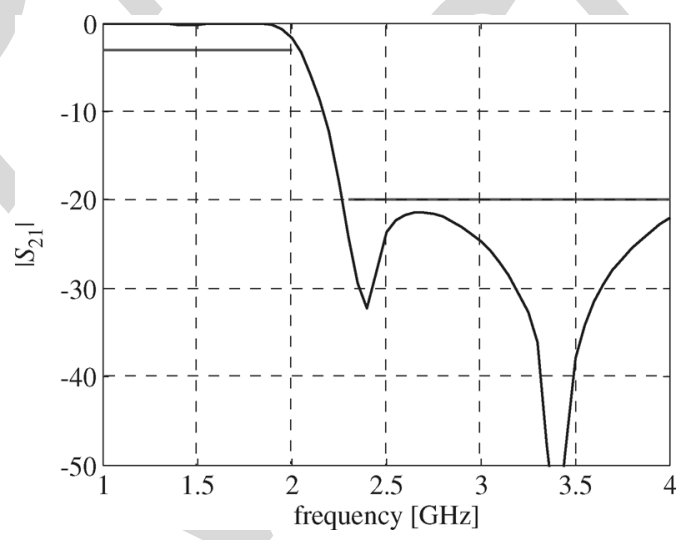

Fig. 18. Low-pass elliptic filter: fine model response at the design found by the space-mapping optimization algorithm with the adaptive response correction (Realization 2).

(transfer function minima) and the response is almost constant at the beginning of the considered frequency range.

Table III shows the optimization results. In this case, the quality of the solution obtained using the standard output space mapping is slightly better than the quality of solution found with the adaptive response correction in terms of specification error value. However, the algorithm with the adaptive response correction requires only three fine model evaluations to find the solution.

Fig. 18 shows the fine model response at the final solution found by the space mapping with Realization 1 of the adaptive response correction.

\section{CONCLUSION}

An adaptive response correction scheme has been presented. The adjustment of the response correction term is related to the changes of the space-mapped coarse model response, which 
occur while optimizing the space-mapping surrogate model. This allows us to alleviate the difficulties of the standard output space mapping that uses a design-variable-independent correction term, which may result in serious distortion of the surrogate model response, and, consequently, deterioration of the performance of the space-mapping algorithm. The robustness of our technique is demonstrated using several microwave design optimization problems.

\section{ACKNOWLEDGMENT}

The authors thank Sonnet Software Inc., Syracuse, NY, for $\boldsymbol{e m}$ and Agilent Technologies, Santa Rosa, CA, for making ADS available.

\section{REFERENCES}

[1] J. W. Bandler, R. M. Biernacki, S. H. Chen, P. A. Grobelny, and R. H. Hemmers, "Space mapping technique for electromagnetic optimization," IEEE Trans. Microw. Theory Tech., vol. 4, no. 12, pp. 536-544, Dec. 1994.

[2] J. W. Bandler, Q. S. Cheng, N. K. Nikolova, and M. A. Ismail, "Implicit space mapping optimization exploiting preassigned parameters," IEEE Trans. Microw. Theory Tech., vol. 52, no. 1, pp. 378-385, Jan. 2004.

[3] J. W. Bandler, Q. S. Cheng, S. A. Dakroury, A. S. Mohamed, M. H. Bakr, K. Madsen, and J. Sondergaard, "Space mapping: The state of the art," IEEE Trans. Microw. Theory Tech., vol. 52, no. 1, pp. 337-361, Jan. 2004.

[4] D. Echeverria and P. W. Hemker, "Space mapping and defect correction," Int. Math. J. Comput. Methods Appl. Math., vol. 5, no. 2, pp. 107-136, 2005.

[5] N. M. Alexandrov and R. M. Lewis, "An overview of first-order model management for engineering optimization," Optim. Eng., vol. 2, no. 4, pp. 413-430, Dec. 2001.

[6] A. J. Booker, J. E. Dennis, Jr., P. D. Frank, D. B. Serafini, V. Torczon, and M. W. Trosset, "A rigorous framework for optimization of expensive functions by surrogates," Struct. Optim., vol. 17, no. 1, pp. 1-13, Feb. 1999.

[7] J. E. Dennis and V. Torczon, "Managing approximation models in optimization," in Multidisciplinary Design Optimization, N. M. Alexandrov and M. Y. Hussaini, Eds. Philadelphia, PA: SIAM, 1997, pp. 330-374.

[8] S. J. Leary, A. Bhaskar, and A. J. Keane, “A knowledge-based approach to response surface modeling in multifidelity optimization," Global Optim., vol. 26, no. 3, pp. 297-319, Jul. 2003.

[9] S. E. Gano, J. E. Renaud, and B. Sanders, "Variable fidelity optimization using a kriging based scaling function," in Proc. 10th AIAA/ISSMO Multidisciplinary Anal. Optim. Conf., Albany, NY, 2004.

[10] T. W. Simpson, J. Peplinski, P. N. Koch, and J. K. Allen, "Metamodels for computer-based engineering design: Survey and recommendations," Eng. Comput., vol. 17, no. 2, pp. 129-150, Jul. 2001.

[11] N. V. Queipo, R. T. Haftka, W. Shyy, T. Goel, R. Vaidynathan, and P. K. Tucker, "Surrogate-based analysis and optimization," Progr. Aerosp. Sci., vol. 41, no. 1, pp. 1-28, Jan. 2005.

[12] M. A. Ismail, D. Smith, A. Panariello, Y. Wang, and M. Yu, "EMbased design of large-scale dielectric-resonator filters and multiplexers by space mapping," IEEE Trans. Microw. Theory Tech., vol. 52, no. 1, pp. 386-392, Jan. 2004.

[13] K.-L. Wu, Y.-J. Zhao, J. Wang, and M. K. K. Cheng, "An effective dynamic coarse model for optimization design of LTCC RF circuits with aggressive space mapping," IEEE Trans. Microw. Theory Tech., vol. 52, no. 1, pp. 393-402, Jan. 2004.

[14] S. Amari, C. LeDrew, and W. Menzel, "Space-mapping optimization of planar coupled-resonator microwave filters," IEEE Trans. Microw. Theory Tech., vol. 54, no. 5, pp. 2153-2159, May 2006.

[15] M. Dorica and D. D. Giannacopoulos, "Response surface space mapping for electromagnetic optimization," IEEE Trans. Magn., vol. 42, no. 4, pp. 1123-1126, Apr. 2006.

[16] J. E. Rayas-Sánchez and V. Gutiérrez-Ayala, "EM-based Monte Carlo analysis and yield prediction of microwave circuits using linear-input neural-output space mapping," IEEE Trans. Microw. Theory Tech., vol. 54, no. 12, pp. 4528-4537, Dec. 2006.
[17] S. J. Leary, A. Bhaskar, and A. J. Keane, "A constraint mapping approach to the structural optimization of an expensive model using surrogates," Optim. Eng., vol. 2, no. 4, pp. 385-398, Dec. 2001.

[18] M. Redhe and L. Nilsson, "Using space mapping and surrogate models to optimize vehicle crashworthiness design," presented at the 9th AIAA/ISSMO Multidisciplinary Anal. Optim. Symp., Atlanta, GA, Sep. 2002, Paper AIAA-2002-5536.

[19] H.-S. Choi, D. H. Kim, I. H. Park, and S. Y. Hahn, "A new design technique of magnetic systems using space mapping algorithm," IEEE Trans. Magn., vol. 37, no. 5, pp. 3627-3630, Sep. 2001.

[20] S. Koziel, J. W. Bandler, and K. Madsen, "A space mapping framework for engineering optimization: Theory and implementation," IEEE Trans. Microw. Theory Tech., vol. 54, no. 10, pp. 3721-3730, Oct. 2006.

[21] S. Koziel and J. W. Bandler, "Space-mapping optimization with adaptive surrogate model," IEEE Trans. Microw. Theory Tech., vol. 55, no. 3, pp. 541-547, Mar. 2007.

[22] S. Koziel, J. W. Bandler, A. S. Mohamed, and K. Madsen, "Enhanced surrogate models for statistical design exploiting space mapping technology," in IEEE MTT-S Int. Microw. Symp. Dig., Long Beach, CA, Jun. 2005, pp. 1609-1612.

[23] S. Koziel, J. W. Bandler, and K. Madsen, "Theoretical justification of space-mapping-based modeling utilizing a data base and on-demand parameter extraction," IEEE Trans. Microw. Theory Tech., vol. 54, no. 12, pp. 4316-4322, Dec. 2006.

[24] S. Koziel and J. W. Bandler, "Microwave device modeling using space-mapping and radial basis functions," in IEEE MTT-S Int. Microw. Symp. Dig., Honolulu, HI, 2007, pp. 799-802.

[25] V. K. Devabhaktuni, B. Chattaraj, M. C. E. Yagoub, and Q.-J. Zhang, "Advanced microwave modeling framework exploiting automatic model generation, knowledge neural networks, and space mapping," IEEE Trans. Microw. Theory Tech., vol. 51, no. 7, pp. 1822-1833, Jul. 2003.

[26] J. E. Rayas-Sánchez, "EM-based optimization of microwave circuits using artificial neural networks: The state-of-the-art," IEEE Trans. Microw. Theory Tech., vol. 52, no. 1, pp. 420-435, Jan. 2004.

[27] J. E. Rayas-Sánchez, F. Lara-Rojo, and E. Martinez-Guerrero, "A linear inverse space-mapping (LISM) algorithm to design linear and nonlinear RF and microwave circuits," IEEE Trans. Microw. Theory Tech., vol. 53, no. 3, pp. 960-968, Mar. 2005.

[28] L. Zhang, J. Xu, M. C. E. Yagoub, R. Ding, and Q.-J. Zhang, "Efficient analytical formulation and sensitivity analysis of neuro-space mapping for nonlinear microwave device modeling," IEEE Trans. Microw. Theory Tech., vol. 53, no. 9, pp. 2752-2767, Sep. 2005.

[29] S. Koziel, J. W. Bandler, and K. Madsen, "Towards a rigorous formulation of the space mapping technique for engineering design," in Proc. Int. Symp. Circuits, Syst., Kobe, Japan, May 2005, pp. 5605-5608.

[30] K. Madsen and J. Søndergaard, "Convergence of hybrid space mapping algorithms," Optim. Eng., vol. 5, no. 2, pp. 145-156, Jun. 2004.

[31] J. W. Bandler, Q. S. Cheng, D. H. Gebre-Mariam, K. Madsen, F. Pedersen, and J. Søndergaard, "EM-based surrogate modeling and design exploiting implicit, frequency and output space mappings," in IEEE MTT-S Int. Microw. Symp. Dig., Philadelphia, PA, Jun. 2003, pp. 1003-1006.

[32] A. R. Conn, N. I. M. Gould, and P. L. Toint, Trust Region Methods, ser. MPS-SIAM Optim. Philadelphia, PA: SIAM, 2000.

[33] N. M. Alexandrov, J. E. Dennis, R. M. Lewis, and V. Torczon, "A trustregion framework for managing the use of approximation models in optimization," Struct. Optim., vol. 15, no. 1, pp. 16-23, 1998.

[34] M. Y. Hsieh and S. M. Wang, "Compact and wideband microstrip bandstop filter," IEEE Microw. Wireless Compon. Lett., vol. 15, no. 7, pp. 472-474, Jul. 2005.

[35] "FEKO User's Manual Suite 5.3" EM Softw. Syst. S.A. (Pty) Ltd., Stellenbosch, South Africa, 2008. [Online]. Available: http://www.feko.info

[36] Agilent Advanced Design System (ADS). ver. 2008, Agilent Technol., Santa Rosa, CA, 2008.

[37] A. Manchec, C. Quendo, J.-F. Favennec, E. Rius, and C. Person, "Synthesis of capacitive-coupled dual-behavior resonator (CCDBR) filters," IEEE Trans. Microw. Theory Tech., vol. 54, no. 6, pp. 2346-2355, Jun. 2006.

[38] W. H. Tu and K. Chang, "Microstrip elliptic-function low-pass filters using distributed elements or slotted ground structure," IEEE Trans. Microw. Theory Tech., vol. 54, no. 10, pp. 3786-3792, Oct. 2006.

[39] em. ver. 11.53, Sonnet Softw. Inc., North Syracuse, NY, 2008. 


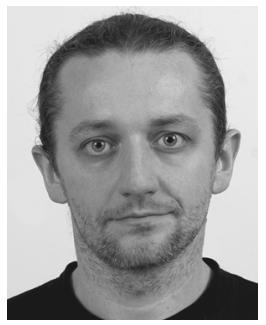

Slawomir Koziel (M'03-SM'07) received the M.Sc. and $\mathrm{Ph} . \mathrm{D}$. degrees in electronic engineering, M.Sc. degrees in theoretical physics and in mathematics, and Ph.D. degree in mathematics from Gdansk University of Technology, Gdansk, Poland, in 1995, 2000, 2000, 2002, and 2003, respectively.

He was a Research Associate with the Department of Electrical and Computer Engineering, McMaster University, Hamilton, ON, Canada. He is currently with the School of Science and Engineering, Reykjavík University, Reykjavík, Iceland. He has authored or coauthored over 120 papers. His research interests include surrogate-based modeling and optimization, space mapping, circuit theory, analog signal processing, evolutionary computation, and numerical analysis.

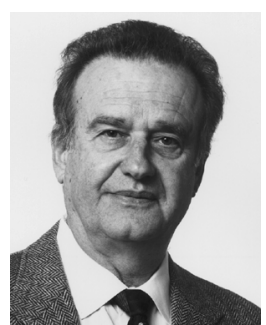

John W. Bandler (S'66-M'66-SM'74-F'78LF'06) studied at Imperial College. He received the B.Sc. (Eng.), Ph.D., and D.Sc. (Eng.) degrees from the University of London, London, U.K., in 1963, 1967, and 1976, respectively.

In 1969, he joined McMaster University, Hamilton, ON, Canada. He is currently a Professor Emeritus. He was President of Optimization Systems Associates Inc., which he founded in 1983, until November 20, 1997, the date of acquisition by the Hewlett-Packard Company. He is President of Bandler Corporation, Dundas, ON, Canada, which he founded in 1997.
Dr. Bandler is a Fellow of several societies including the Royal Society of Canada. He was the recipient of the 2004 IEEE Microwave Theory and Techniques Society (IEEE MTT-S) Microwave Application Award.

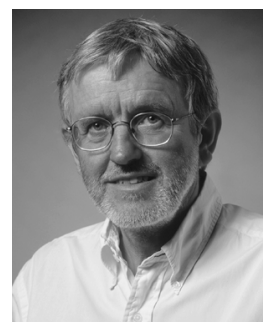

Kaj Madsen was born in [City,] Denmark, in 1943. He undertook his basic education in mathematics at the University of Aarhus, Aarhus, Denmark. He received the Dr.Techn. degree from the Technical University of Denmark (DTU), Lyngby, Copenhagaen, Denmark, in [Year].

He has spent his career with DTU and Copenhagen University. He is currently a Full Professor with DTU. He was a main force in creating the Department of Informatics and Mathematical Modelling, DTU, which includes computer science, communications, and applied mathematics, and since 1995, has headed the department. His primary research interests are nonlinear optimization including space-mapping techniques and global optimization using interval analysis. 